\title{
Improved Monte Carlo Glauber predictions at present and future nuclear colliders
}

\author{
Constantin Loizides \\ Oak Ridge National Laboratory, Oak Ridge, Tennessee 37830, USA \\ Jason Kamin \\ University of Illinois at Chicago, Chicago, Illinois 60607, USA \\ David d'Enterria \\ CERN, Geneva, Switzerland
}

(Received 23 November 2017; published 23 May 2018)

\begin{abstract}
We present the results of an improved Monte Carlo Glauber (MCG) model of relevance for collisions involving nuclei at center-of-mass energies of the BNL Relativistic Heavy Ion Collider $\left(\sqrt{s_{N N}}=0.2 \mathrm{TeV}\right)$, CERN Large Hadron Collider (LHC) $\left(\sqrt{s_{N N}}=2.76-8.8 \mathrm{TeV}\right)$, and proposed future hadron colliders $\left(\sqrt{s_{N N}} \approx 10-63 \mathrm{TeV}\right)$. The inelastic $p p$ cross sections as a function of $\sqrt{s_{N N}}$ are obtained from a precise data-driven parametrization that exploits the many available measurements at LHC collision energies. We describe the nuclear density of a lead nucleus with two separated two-parameter Fermi distributions for protons and neutrons to account for their different densities close to the nuclear periphery. Furthermore, we model the nucleon degrees of freedom inside the nucleus through a lattice with a minimum nodal separation, combined with a "recentering and reweighting" procedure, that overcomes some limitations of previous MCG approaches. The nuclear overlap function, number of participant nucleons and binary nucleon-nucleon collisions, participant eccentricity and triangularity, overlap area, and average path length are presented in intervals of percentile centrality for lead-lead $(\mathrm{PbPb})$ and proton-lead $(p \mathrm{~Pb})$ collisions at all collision energies. We demonstrate for collisions at $\sqrt{s_{N N}}=5.02 \mathrm{TeV}$ that the central values of the Glauber quantities change by up to $7 \%$ in a few bins of reaction centrality, due to the improvements implemented, though typically they remain within the previously assigned systematic uncertainties, while their new associated uncertainties are generally smaller (mostly below 5\%) at all centralities than for earlier calculations. Tables for all quantities versus centrality at present and foreseen collision energies involving $\mathrm{Pb}$ nuclei, as well as for collisions of $\mathrm{XeXe}$ at $\sqrt{s_{N N}}=5.44 \mathrm{TeV}$, and $\mathrm{AuAu}$ and $\mathrm{CuCu}$ at $\sqrt{s_{N N}}=0.2 \mathrm{TeV}$, are provided. The source code for the improved Monte Carlo Glauber model is made publicly available.
\end{abstract}

DOI: 10.1103/PhysRevC.97.054910

\section{INTRODUCTION}

The interpretation of many results measured in high-energy heavy-ion collisions relies on the use of a model of the initial matter distribution resulting from the overlap of the two colliding nuclei at a given impact parameter $b$. Indeed, quantities such as (i) the centrality dependence, expressed by the number of participating nucleons in the collision, $N_{\text {part }}(b)$, of any observable, (ii) the nuclear overlap function $T_{A A}(b)$ or the number of binary nucleon-nucleon collisions, $N_{\text {coll }}(b)$, used to derive the nuclear modification factor $\left(R_{A A}\right)$ from the ratio of $A A$ over $p p$ spectra, (iii) the elliptic and triangular flow parameters $v_{2}$ and $v_{3}$ normalized by the eccentricity $\varepsilon_{2}(b)$ and triangularity $\varepsilon_{3}(b)$ of the overlap region, and the average (iv) surface area $A_{\mathrm{T}}(b)$ and (v) path length $L(b)$ of the interaction region all depend on a realistic model of the collision geometry [1].

Published by the American Physical Society under the terms of the Creative Commons Attribution 4.0 International license. Further distribution of this work must maintain attribution to the author(s) and the published article's title, journal citation, and DOI.
The standard method employed in high-energy heavy-ion collisions describes the initial transverse shape of the nuclei in terms of two-parameter Fermi $(2 \mathrm{pF})$ distributions (also often called Wood-Saxon distributions) with parameters half-density radius $R$ and diffusivity $a$ obtained from fits to elastic lepton-nucleus data [2,3], and determines the underlying multinucleon interactions in the overlap area between the nuclei through a Glauber eikonal approach [4]. In the Monte Carlo Glauber (MCG) models (e.g., Refs. [5-10]), individual nucleons are sampled event-by-event from the underlying $2 \mathrm{pF}$ distributions and the collision properties are calculated by averaging over multiple events. However, neutron-rich nuclei such as ${ }^{208} \mathrm{~Pb}$ may have differing proton and neutron density distributions at the nuclear periphery. Indeed, measurements have recently been able to extract the neutron profile of several nuclei that show differences with respect to their proton distribution $[11,12]$, and various works have already studied its impact on different isospin-dependent observables in nuclear collisions [13-15].

In this article, we present the results of improved Glauber Monte Carlo model calculations for $N_{\text {coll }}(b), N_{\text {part }}(b), T_{A A}(b)$, $\varepsilon_{2}(b), \varepsilon_{3}(b), A_{\mathrm{T}}(b)$, and $L(b)$ in $\mathrm{PbPb}$ and $p \mathrm{~Pb}$ collisions at the Large Hadron Collider (LHC) $\left(\sqrt{s_{N N}}=2.76,5.02,5.5,8.16\right.$, 
and $8.8 \mathrm{TeV})$, High-Energy LHC $\left(\sqrt{s_{N N}}=10.6,17 \mathrm{TeV}\right)$, and Future Circular Collider (FCC) $\left(\sqrt{s_{N N}}=39\right.$ and $\left.63 \mathrm{TeV}\right)$ [16] energies, by considering for the first time separated transverse profiles for protons and neutrons in the lead nucleus. The corresponding values for the inelastic $p p$ cross section are obtained from a data-driven parametrization with reduced uncertainties thanks to the many available measurements at LHC collision energies. The nucleon degrees of freedom inside a nucleus are modeled using a lattice with a minimum nodal separation that mimics hard-core repulsion between nucleons without distorting the nuclear density. Residual small distortions in the generated nuclear densities resulting from adjusting the nucleon center of mass (c.m.) with that of the nucleus are overcome by reweighting the original nuclear density. We exemplify for collisions at $\sqrt{s_{N N}}=5.02 \mathrm{TeV}$ that the central values of $N_{\text {coll }}(b), N_{\text {part }}(b), T_{A A}(b)$, and $\varepsilon_{2}(b)$ change due to the inclusion of the separated proton and neutron transverse distributions, but typically remain within the previously assigned systematic uncertainties. Their new associated uncertainties are generally smaller than for earlier calculations except for mid-peripheral events where they are slightly larger in some cases. Tables for all quantities versus centrality at present and foreseen collision energies involving $\mathrm{Pb}$ nuclei are provided. Results for other studied systems, such as $\mathrm{AuAu}$ and $\mathrm{CuCu}$ collisions at $\sqrt{s_{N N}}=0.2 \mathrm{TeV}$ and XeXe collisions at $\sqrt{s_{N N}}=5.44 \mathrm{TeV}$, are provided also for completeness. As for previous versions of the model, the source code for "TGlauberMC" (version 3.0) has been made publicly available at HepForge [17].

The paper is organized as follows: Section II describes the basic quantities of interest computed in the article. Section III presents a parametrization of the c.m. energy dependence of the nucleon inelastic cross section $\left(\sigma_{N N}\right)$ based on existing protonproton $(p p)$ and proton-antiproton $(p \bar{p})$ data. Section IV introduces the basic details of the MCG calculation. Section V discusses the improvements of the MCG modeling, namely, using a more realistic nuclear matter density with separated proton and neutron profiles (Sec. VA), incorporating a minimum internucleon separation without distorting the nuclear profile (Sec. VB), reweighting the nuclear density to compensate residual distortions introduced by the nucleon center-of-mass recentering (Sec. VC), and using a more precise parametrization of the $\sigma_{N N}(\mathrm{Sec}$. V D). Section VI presents the results of the improved MCG calculation and Sec. VII summarizes our main conclusions. Appendix A illustrates the difference between an optical and a Monte Carlo Glauber calculation. Appendix B briefly discusses the inclusion of subnucleonic degrees of freedom in the MCG calculation. Appendix $\mathrm{C}$ provides an updated user's guide for running the publicly available MCG code. Appendix D provides tables with calculated quantities for all relevant collision energies involving $\mathrm{Pb}$ nuclei, including $\mathrm{XeXe}$ collisions at $\sqrt{s_{N N}}=5.44 \mathrm{TeV}$ as well as $\mathrm{AuAu}$ and $\mathrm{CuCu}$ collisions at $\sqrt{s_{N N}}=0.2 \mathrm{TeV}$.

\section{GLAUBER FORMALISM}

The standard procedure to determine the transverse overlap area, and other derived quantities in a generic proton-nucleus $(p A)$ or nucleus-nucleus collision $(A B)$ at impact parameter $b$, is based on a simple Glauber multiscattering eikonal model that assumes straight-line trajectories of the nucleons from the two colliding nuclei [4]. A review that describes the basic formalism can be found in Ref. [1], of which we briefly summarize the main concepts here.

To simplify the mathematical description, the reaction plane of the two colliding nuclei, i.e., the plane defined by the impact parameter and the beam direction, is given by the $x$ and $z$ axes, while the transverse plane is given by the $x$ and $y$ axes. The collision impact parameter $b$ is distributed assuming $d N / d b \propto b$, and the centers of the nuclei are shifted to $\left(-\frac{b}{2}, 0,0\right)$ and $\left(\frac{b}{2}, 0,0\right)$.

In "optical" Glauber calculations a smooth nuclear matter density, $\rho$, for each nucleus is used and properties of the reaction zone and all derived quantities are analytically calculated. In Monte Carlo based approaches individual nucleons are distributed for each nucleus according to $\rho$ in an eventby-event basis and collision properties as well as derived quantities are calculated by averaging over multiple events. In both cases, following the eikonal ansatz, the nucleons are assumed to move in straight trajectories along the beam axis. The nuclear reaction is modeled by successive independent interactions between two nucleons from different nuclei, where the interaction strength between two nucleons is typically modeled using the nucleon-nucleon inelastic cross section $\left(\sigma_{N N}\right)$ in the transverse plane. The transverse positions of nucleons are assumed to be constant during the short passage time of the two high-energy nuclei, while their longitudinal coordinate does not play a role in the calculation.

The optical calculations are based on the thickness function of a nucleus, which quantifies the transverse nucleon density as $T(x, y)=\int \rho(x, y, z) d z$, which is usually normalized to the number of nucleons in the nucleus $A$. The nuclear overlap function of nuclei $A$ and $B$ colliding at impact parameter $b, T_{A B}(b)$, can then be expressed as the convolution of the corresponding thickness functions of $A$ and $B$,

$$
\begin{aligned}
T_{A B}(b) & =\int \rho_{\text {coll }}(x, y, b) d x d y \\
& =\int T_{\mathrm{A}}\left(x-\frac{b}{2}, y\right) T_{\mathrm{B}}\left(x+\frac{b}{2}, y\right) d x d y,
\end{aligned}
$$

usually normalized so that $\int T_{A B}(b) b d b=A B$.

The number of nucleons in the target and projectile nuclei that interacted at least once in a collision at impact parameter $b$ is called the number of participants (or "wounded nucleons") and is calculated as [18,19]

$$
\begin{aligned}
N_{\text {part }}(b)= & \int \rho_{\text {part }}(x, y, b) d x d y \\
= & A \int T_{\mathrm{A}}^{-}\left(1-\left[1-\sigma_{N N} T_{\mathrm{B}}^{+}\right]^{B}\right) d x d y \\
& +B \int T_{\mathrm{B}}^{+}\left(1-\left[1-\sigma_{N N} T_{\mathrm{A}}^{-}\right]^{A}\right) d x d y
\end{aligned}
$$

with $T_{\mathrm{X}}^{ \pm} \equiv T_{\mathrm{X}}\left(x \pm \frac{b}{2}, y\right)$. Similarly, the total number of binary nucleon-nucleon collisions at impact parameter $b$ is calculated as

$$
N_{\mathrm{coll}}(b)=\sigma_{N N} \int \rho_{\mathrm{coll}}(x, y, b) d x d y=\sigma_{N N} T_{A B}(b) .
$$


Hence, the nuclear overlap function, $T_{A B}(b)=N_{\text {coll }}(b) / \sigma_{N N}$, can be thought of as the nucleon-nucleon luminosity (reaction rate per unit cross section) in an $A B$ collision at a given impact parameter $b$.

MCG calculations obtain the quantities (2) and (3) by simply counting either the number of nucleons that interacted at least once $\left(N_{\text {part }}\right)$, or the total number of individual nucleonnucleon collisions $\left(N_{\text {coll }}\right)$, where the collisions between the nucleons of the two incoming nuclei are determined by a $\sigma_{N N}$-dependent interaction probability in the transverse plane.

The second moment, also called eccentricity [20], the third moment, also called triangularity [21], and higher moments [22] of the collision region at impact parameter $b$, which are used to characterize the initial geometrical shape, are given by

$$
\varepsilon_{n}(b)=\frac{\left\langle r^{n} \cos (n \phi-n \psi)\right\rangle}{\left\langle r^{n}\right\rangle},
$$

where $n$ denotes the moment ( $n=2$ for eccentricity, $n=3$ for triangularity), $r=\sqrt{x^{2}+y^{2}}$, and $\psi=\tan ^{-1} \frac{y}{x}$. The averages are performed by considering the central positions of either participant nucleons or binary nucleon-nucleon collisions, or of an admixture of the two.

The effective transverse overlap area between the two nuclei is often taken to be proportional to the widths of the participant distributions,

$$
A_{\perp}(b) \propto \sqrt{\left\langle x^{2}\right\rangle\left\langle y^{2}\right\rangle},
$$

where the averages are taken over participant nucleons. There is no commonly accepted definition of the absolute normalization of the overlap area. Historically, either $\pi$ [23] or $4 \pi$ [24] has been used, where the latter essentially coincides with the geometrical overlap area of two uniform disks. Recently, it was proposed to directly calculate the area in the MCG by evaluating the area of wounded nucleons with a fine-grained grid [10].

The average path length through a static medium with a density parametrized with $\rho_{\text {part }}$ can be calculated using

$$
L(b)=\frac{\int l \rho_{\text {part }}\left(x_{0}+l \cos \phi_{0}, y_{0}+l \sin \phi_{0}, b\right) d l d P_{0}}{0.5 \int \rho_{\text {part }}\left(x_{0}+l \cos \phi_{0}, y_{0}+l \sin \phi_{0}, b\right) d l d P_{0}},
$$

where the initial point $\left(x_{0}, y_{0}\right)$ is usually distributed according to $\rho_{\text {coll }}$ and the azimuthal direction $\phi_{0}$ uniformly $[25,26]$.

The total inelastic cross sections for $p A$ or $A B$ collisions are

$$
\sigma_{p A}=2 \pi \int b\left[1-e^{-\sigma_{N N} T_{\mathrm{A}}(b)}\right] d b
$$

and

$$
\sigma_{A B}=2 \pi \int b\left[1-e^{-\sigma_{N N} T_{A B}(b)}\right] d b .
$$

MCG calculations obtain the cross sections by simply multiplying the fraction of accepted events with $\pi b_{\max }^{2}$, where $b_{\max }$ is the maximum generated impact parameter (usually $20 \mathrm{fm}$ ).

Observables are often studied in intervals of cross sections, called "centrality percentiles," whose experimental ranges are typically obtained by ordering the events according to their particle multiplicity or transverse energy and, in the case of $A A$ collisions, can be translated into equivalent ranges of

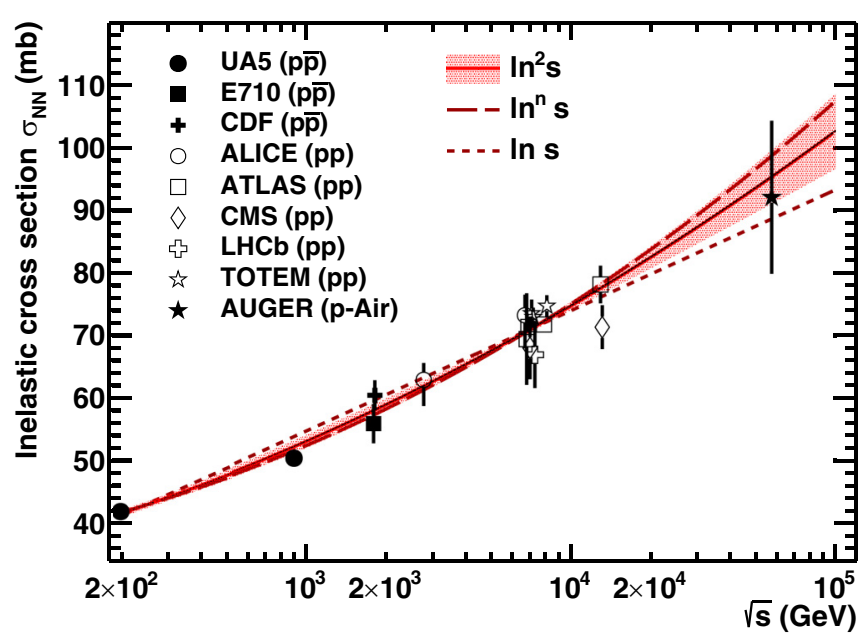

FIG. 1. Inelastic $p p$ cross section as a function of c.m. energy in the range $\sqrt{s}=0.2-100 \mathrm{TeV}$. Experimental data points at various colliders and cosmic-ray energies from UA5 [29], E710 [30,31], CDF [32,33], ALICE [34], ATLAS [35-38], CMS [39,40], LHCb [41], TOTEM [42-44], and AUGER [45]. Fits of $\ln s, \ln ^{2} s$ and $\ln ^{n} s$ to the data are shown (for details see text).

impact parameter (see, e.g., Ref. [27]). Instead of reporting results as a function of centrality percentiles, often the mean number of participants in the centrality interval is used, which, like all quantities in a Glauber calculation, can be obtained by performing the calculation over their respective impact parameter range.

\section{PARAMETRIZATION OF THE INELASTIC NUCLEON-NUCLEON CROSS SECTION}

A fundamental ingredient of any Glauber calculation is the inelastic nucleon-nucleon cross section, $\sigma_{N N}$, at the same c.m. energy $\sqrt{s_{N N}}$ of the nuclear collision under consideration. The value of $\sigma_{N N}$ includes particle production contributions from both (semi)hard parton-parton scatterings, computable above a given $p_{\mathrm{T}} \approx 2 \mathrm{GeV}$ cutoff by perturbative QCD approaches, as well as from softer "peripheral" scatterings of diffractive nature, with a scale not very far from $\Lambda_{\mathrm{QCD}} \approx 0.2 \mathrm{GeV}$. Today, $\sigma_{N N}$ cannot be computed from first-principles QCD calculations (although future developments in lattice QCD computations could improve this situation) and one resorts to phenomenological approaches to fit the experimental data and predict their evolution as a function of $\sqrt{s_{N N}}$ [28]. At high c.m. energies, above a few tens of $\mathrm{GeV}, p p$ and $p \bar{p}$ (as well as $n n$ and $n p$ ) collisions all feature the same inelastic cross sections. Any potential differences due to their different valence-quark structure are increasingly irrelevant, and all existing experimental measurements can be combined to extract $\sigma_{N N}$. The $\sqrt{s}$ dependence of the inelastic cross section $\sigma_{N N}$ is shown in Fig. 1 for all the available data from $p \bar{p}$ and $p p$ colliders, and the AUGER result at $\sqrt{s}=57 \mathrm{TeV}$ derived from cosmic-ray data [45]. We include $p \bar{p}$ measurements from UA5 [29] at $\sqrt{s}=200$ and $900 \mathrm{GeV}$, E710 [30,31] and CDF [32,33] at $\sqrt{s}=1.8 \mathrm{TeV}$, as well as $p p$ results from ALICE at $7 \mathrm{TeV}$ [34], ATLAS at 7, 8, and $13 \mathrm{TeV}$ [35-38], CMS at 7 and 
TABLE I. Fit values and $\chi^{2} / N_{\text {dof }}$ for the collision-energy dependence of $\sigma_{N N}$ parametrized by Eq. (9) and displayed in Fig. 1.

\begin{tabular}{lcccc}
\hline \hline Type & $A$ & $B$ & $n$ & $\chi^{2} / N_{\text {dof }}$ \\
\hline $\ln s$ & $-3.33 \pm 1.58$ & $4.195 \pm 0.103$ & 1 (fixed) & 1.52 \\
$\ln ^{2} s$ & $25.0 \pm 0.9$ & $0.146 \pm 0.004$ & 2 (fixed) & 0.97 \\
$\ln ^{n} s$ & $29.8 \pm 4.7$ & $0.038 \pm 0.060$ & $2.43 \pm 0.50$ & 0.98 \\
\hline \hline
\end{tabular}

$13 \mathrm{TeV}[39,40], \mathrm{LHCb}$ at $7 \mathrm{TeV}$ [41], and TOTEM at 7 and $8 \mathrm{TeV}$ [42-44]. The experimental $\sigma_{N N}$ values plotted are either obtained (i) from the subtraction $\sigma_{\text {inel }}=\sigma_{\text {tot }}-\sigma_{\text {el }}$, where $\sigma_{\text {tot }}$ and $\sigma_{\mathrm{el}}$ have been accurately measured in dedicated forward Roman pot detectors (TOTEM [42-44] and ALFA [36-38]), or (ii) from measurements of inelastic particle production data in the central detectors collected with "minimum bias" triggers. The latter measurements are less accurate than the former, as they require an extrapolation, dominated by diffractive contributions, to forward regions of phase space not covered by detectors and therefore have larger uncertainties.

The collision-energy dependence of $\sigma_{N N}$ has been fit to the parametrization

$$
\sigma_{N N}(s)=A+B \ln ^{n}(s),
$$

where $n$ was fixed to either $n=1$ or $n=2$, or otherwise left free in the fit. The values and $\chi^{2} / N_{\text {dof }}$ for the three cases are given in Table I. The $n=2$ case, which represents the asymptotic $\sqrt{s}$ dependence expected to saturate the Froissart bound [46], is used as the central value for the interpolation (and extrapolation) of $\sigma_{N N}$ versus $\sqrt{s}$, listed in Table II for relevant LHC and FCC energies. The difference (normalized by 2.4 to account for the full width at half maximum) of the

TABLE II. Values of the nucleon-nucleon inelastic cross section $\sigma_{N N}$ extracted from the $\ln ^{2}$ fit, with the uncertainties estimated from the difference of the $\ln s$ and $\ln ^{n} s(n=2.43)$ fits at collision energies $\sqrt{s}$ relevant for RHIC, LHC, and FCC.

\begin{tabular}{lr}
\hline \hline$\sqrt{s}(\mathrm{TeV})$ & $\sigma_{N N}(\mathrm{mb})$ \\
\hline 0.2 & $41.6 \pm 0.6$ \\
0.9 & $52.2 \pm 1.0$ \\
2.76 & $61.8 \pm 0.9$ \\
5.02 & $67.6 \pm 0.6$ \\
5.44 & $68.4 \pm 0.5$ \\
5.5 & $68.5 \pm 0.5$ \\
7 & $70.9 \pm 0.4$ \\
8 & $72.3 \pm 0.5$ \\
8.16 & $72.5 \pm 0.5$ \\
8.8 & $73.3 \pm 0.6$ \\
10.6 & $75.3 \pm 0.7$ \\
13 & $77.6 \pm 1.0$ \\
14 & $78.4 \pm 1.1$ \\
17 & $80.6 \pm 1.5$ \\
27 & $86.0 \pm 2.4$ \\
39 & $90.5 \pm 3.3$ \\
63 & $96.5 \pm 4.6$ \\
100 & $102.6 \pm 6.0$ \\
\hline \hline
\end{tabular}

so-derived $\sigma_{N N}$ values from those obtained for $n=1,2.43$ is assigned as systematic uncertainty (shown as a band in Fig. 1). The resulting cross section at $100 \mathrm{TeV}$ of $\sigma_{N N}=$ $102.6 \pm 6.0 \mathrm{mb}$ is consistent with the value $105.1 \pm 2.0 \mathrm{mb}$, obtained from the average of various model calculations [28]. The value extracted for the top Relativistic Heavy Ion Collider (RHIC) energy of $\sqrt{s_{N N}}=0.2 \mathrm{TeV}$ is $41.6 \pm 0.6 \mathrm{mb}$ and is consistent with the typically used value of $42 \pm 3 \mathrm{mb}$ [23].

Other more complicated functional forms were also tried, motivated by the ansatz used by the COMPETE collaboration [47], such as $\sigma_{N N}(\sqrt{s})=A+B \ln ^{2}(s)+C s^{-\eta}$, and $\sigma_{N N}(\sqrt{s})=A+B \ln ^{2}(s)+D \ln (s)$. The corresponding fits resulted in $A=24.4 \pm 1.4, B=0.1008 \pm 0.1537, C=$ $1.454 \pm 1.768$, and $\eta=0.131 \pm 0.0180$ with $\chi^{2} / N_{\text {dof }}=$ 1.09 , and $A=39.7 \pm 1.4, B=0.2212 \pm 0.0708$, and $D=$ $-2.154 \pm 2.035$ with $\chi^{2} / N_{\text {dof }}=0.96$, respectively. Both parametrizations turned out to be numerically close to Eq. (9) for $n \approx 2.43$ as determined by the simpler $\ln ^{n} s$ fit.

From the obtained values of $\sigma_{N N}$, one can then easily derive the corresponding proton-nucleus and nucleus-nucleus inelastic collisions making use of Eqs. (7) and (8). The computed $\sigma_{p A}$ and $\sigma_{A B}$ results for all relevant collision systems in this work are listed in Table $\mathrm{V}$. The Glauber calculation gives $\sigma_{\mathrm{PbPb}}^{\mathrm{MC}}=$ $7.57 \pm 0.03 \mathrm{~b}$ and $\sigma_{p \mathrm{~Pb}}^{\mathrm{MC}}=2.08 \pm 0.01 \mathrm{~b}$ for the hadronic $\mathrm{PbPb}$ and $p \mathrm{~Pb}$ cross sections, in good agreement with the measured values of $\sigma_{\mathrm{PbPb}}=7.7 \pm 0.6 \mathrm{~b}$ at $\sqrt{s_{N N}}=2.76 \mathrm{TeV}$ [48] and $\sigma_{p \mathrm{~Pb}}=2.06 \pm 0.08 \mathrm{~b}$ [49] as well as $\sigma=2.10 \pm 0.07$ [50] at $\sqrt{s_{N N}}=5.02 \mathrm{TeV}$, respectively.

\section{DETAILS OF THE MCG CALCULATION}

The implementation of the MCG calculation is described in detail in Refs. [6,7]. It consists of two steps: first, constructing the nuclei, and second, colliding the nuclei.

To construct a nucleus, the position of each nucleon is determined according to a probability density function usually taken from measurements of the charge density distribution of the nucleus [2,3]. For spherical nuclei, the nucleon positions can be determined in polar coordinates with a uniform distribution for the azimuthal and polar angles, coupled with a $2 \mathrm{pF}$ distribution in the radial direction:

$$
\rho(r)=\frac{\rho_{0}}{1+\exp [(r-R) / a]},
$$

where $\rho_{0}$ is a normalization constant so that $\int d^{3} r \rho(r)=1$. The half-density or central radius $R$ describes the mean location of the nucleus area (i.e., $R$ is indicative of the extension of the bulk part of the density distribution). The diffusivity parameter $a$ describes the tail of the density profile. Values for $\mathrm{Pb}$ nuclei are listed in Table IV, while a complete list of parameters for other nuclei can be found in Ref. [7]. To mimic a hard-core repulsion potential between nucleons, a minimum internucleon separation $\left(d_{\mathrm{min}}\right)$ of usually $0.4 \mathrm{fm}$ between their centers is enforced when sampling the positions of the nucleons inside a nucleus. To ensure that the center of mass of each constructed nucleus is at $(0,0,0)$, the nucleons are individually "recentered" through a procedure discussed in more detail later.

To simulate the collision, the centers of the nuclei are then shifted to $(-b / 2,0,0)$ and $(b / 2,0,0)$. The collision of two 
TABLE III. Parameters with corresponding uncertainties for the traditional and improved MCG model used to compute Glauber quantities for nuclear collisions at a reference c.m. energy of $\sqrt{s_{N N}}=$ $5.02 \mathrm{TeV}$.

\begin{tabular}{lcc}
\hline \hline MCG model & Traditional & Improved \\
\hline Density for $\mathrm{Pb}$ & Charge, $2 \mathrm{pF}$ ("Pb") & Point, $\mathrm{D} 2 \mathrm{pF}$ ("Pbpnrw") \\
$N N$ separation (fm) & $d_{\min }=0.4 \pm 0.4$ & $d_{\text {node }}=0.4 \pm 0.4$ \\
$\sigma_{N N}(\mathrm{mb})$ & $70 \pm 5$ & $67.6 \pm 0.5$ \\
Recentering & Shift & $d_{\max }=0.1 \mathrm{fm}+$ reweight \\
TGlauberMC & $\equiv \mathrm{v} 2 . \mathrm{x}$ & $\equiv \mathrm{v} 3 . \mathrm{x}$ \\
\hline \hline
\end{tabular}

nuclei is then modeled by assuming that the nucleons of each nucleus travel in a straight line along the beam axis in the transverse plane (eikonal approximation), ignoring their longitudinal coordinates in the calculation. The impact parameter of the collision is chosen randomly from $d N / d b \propto b$ up to some large maximum $b_{\max } \simeq 20 \mathrm{fm}$, chosen to be significantly greater than twice the nuclear radius. Two nucleons from different nuclei are usually assumed to collide if their relative transverse distance is less than a diameter given by

$$
D=\sqrt{\sigma_{N N} / \pi}
$$

which geometrically parametrizes the interaction strength of two nucleons for a given value of $\sigma_{N N}$. If no nucleon-nucleon collision is registered for any pair of nucleons, then no nucleusnucleus collision occurred. Counters for determination of the total (geometric) cross section are updated accordingly. The inelastic nucleon-nucleon cross section $\sigma_{N N}$ is either directly taken from measurements in $p p$ collisions, or extracted from interpolations of the available data, as explained in Sec. III.

Constructing the nucleus is a principal ingredient of the MCG model and the dominant source of systematic uncertainties in the Glauber quantities, in particular after reducing the uncertainties of the interpolated $\sigma_{N N}$ values. In the following, we discuss improvements of the MCG model aiming at achieving a more accurate baseline description with reduced systematic uncertainties. The new results are labeled improved MCGand discussed in detail in the next section. To compare with previous baseline results, we compare the results of our new calculations with those from a set of traditional parameters of the MCG model, typically used in previous studies [10,27,51], given by $\sigma_{N N}=64 \mathrm{mb}$ for $\sqrt{s_{N N}}=2.76 \mathrm{TeV}$ and $\sigma_{N N}=70$ $\mathrm{mb}$ for $\sqrt{s_{N N}}=5.02 \mathrm{TeV}$ with an uncertainty of $\pm 5 \mathrm{mb}$, with charge radius and diffusivity of the nuclear density profile varied within their measured $1 \sigma$ uncertainties, and minimum internucleon separation distance varied by $100 \%$, i.e., between 0 and $0.8 \mathrm{fm}$. The algorithmic definitions, as well as central values and uncertainties of the parameters, for the traditional and improved MCG setups are summarized in Table III.

\section{IMPROVEMENTS OF THE MCG MODELING}

\section{A. Nuclear matter density}

The nuclear density parameters used for the $2 \mathrm{pF}$ distributions are typically taken from the Atomic Data and Nuclear Data Tables (ADND) [2,3,52-54]. They are extracted via
Coulomb scattering in electron-nucleus and muon-nucleus measurements and therefore dominantly probe the charge density of the nucleus. Since ${ }^{208} \mathrm{~Pb}$ is a doubly-magicnucleus (both the number of protons, 82, and number of neutrons, 126, are arranged in fully closed energy shells), it is rather immune to shape deformations, and hence its charge density is well described by a $2 \mathrm{pF}$ distribution, with $R$ and $a$ determined to within $1 \%$ and $2 \%$, respectively. Traditionally, the values for ${ }^{207} \mathrm{~Pb}$ from Ref. [3] are used instead of those for ${ }^{208} \mathrm{~Pb}$ from Ref. [2] when modeling ${ }^{208} \mathrm{~Pb}$ in MCG calculations. ${ }^{1}$

However, since the MCG uses the charge density to place the central locations of each nucleon, a preferred representation is the point density distribution, which parametrizes the $2 \mathrm{pF}$ function for the centers of the nucleons. Transforming from the charge to point distribution involves parameter unfolding, which is performed using the proton root-mean-square (rms) charge radius $\sqrt{\left\langle r^{2}\right\rangle}=0.875 \mathrm{fm}$ [55] via the prescription given in Refs. $[11,56]$. The point density $2 \mathrm{pF}$ parameter values are slightly smaller than the charge density ones due to the proton's finite spatial extension. The corresponding uncertainties on the proton radius density $R_{p}$ have become smaller over the years and are now below $0.5 \%$ [52-54]. However, the diffusivity parameter for protons, $a_{p}$, is no longer quoted in the more recent ADND tables. Moreover, it has been shown that at very large radii (distances greater than $\approx R+3 a$ ) the $2 \mathrm{pF}$ parametrization begins to fail because the measured density falls off faster than a Woods-Saxon distribution [57]. This observation can be modeled by letting the diffusivity parameter shrink with increasing $r$, and while the authors provide $a(r)$ for ${ }^{40} \mathrm{Ca}$ and ${ }^{48} \mathrm{Ca}$, they do not provide it for ${ }^{208} \mathrm{~Pb}$. Thus, we maintain the constant- $a 2 \mathrm{pF}$ form and, in turn, sustain the traditional relatively large uncertainty on the diffusivity parameter of about $2 \%( \pm 0.01 \mathrm{fm})$.

Using the nucleon point density distribution leads to a more realistic placement of the nucleons. However, there is evidence that the proton and neutron distributions may not be exactly the same at the surface of heavy stable nuclei [58]. This effect is particularly important in neutron-rich nuclei, such as ${ }^{208} \mathrm{~Pb}$ with a neutron excess of $N / Z \approx 1.5$. Protons near the center of the nucleus feel electrostatic repulsion from all directions, resulting in an electrostatic equilibrium and a constant charge density. However, at $r \gtrsim 6 \mathrm{fm}$, where the nucleon density begins to drop, the outermost protons need additional "skin" or "halo" neutrons in the periphery to counteract the outward Coulomb repulsion and maintain a sufficient nuclear surface tension, thereby increasing the overall binding energy.

To extract the $2 \mathrm{pF}$ parameters for neutrons, the Crystal Ball collaboration has performed a measurement via coherent pion photoproduction [12] while the Low Energy Proton Ring (LEAR) at CERN has investigated antiproton-nucleus interactions coupled with radiochemistry techniques [11]. The former extracts neutron point density parameters of $R_{\mathrm{n}}=6.70 \pm 0.03$

\footnotetext{
${ }^{1}$ There is no clear reason for that, and we speculate that it may simply be an oversight because the ${ }^{208} \mathrm{~Pb}$ parameters were only collected in the earliest, but not in the later, ADND publications. In any case, the two sets of parameters are essentially the same, as can be seen in Table IV.
} 
TABLE IV. Nuclear density parameters of $\mathrm{Pb}$ for charge and point density distributions. For the neutron point density, the values are averaged as explained in the text. The name of the corresponding profile in the TGlauberMC implementation [17] is also listed.

\begin{tabular}{lccc}
\hline \hline & Name & $R(\mathrm{fm})$ & $a(\mathrm{fm})$ \\
\hline \multicolumn{4}{c}{ Charge density } \\
${ }^{207} \mathrm{~Pb} \mathrm{[3]}$ & $\mathrm{Pb}$ & $6.620 \pm 0.060$ & $0.546 \pm 0.010$ \\
${ }^{208} \mathrm{~Pb} \mathrm{[2]}$ & $\mathrm{Pb}^{*}$ & $6.624 \pm 0.035$ & $0.549 \pm 0.008$ \\
\multicolumn{3}{c}{ Point density } \\
${ }^{208} \mathrm{~Pb}$ & $\mathrm{Pbpn}$ & $6.68 \pm 0.02$ & $0.447 \pm 0.01$ \\
proton [11] & \multicolumn{4}{c}{$6.69 \pm 0.03$} & $0.560 \pm 0.03$ \\
neutron [11,12] & & 6
\end{tabular}

(stat.) fm and $a_{\mathrm{n}}=0.55 \pm 0.01$ (stat.) ${ }_{-0.03}^{+0.02}$ (syst.) fm, while the latter reports comparable values of $R_{\mathrm{n}}=6.684 \pm 0.020$ (stat.) fm and $a_{\mathrm{n}}=0.571 \mathrm{fm}$. These data favor the peripheral neutron distribution in the form of a neutron "halo" rather than a neutron "skin"; i.e., the neutron distribution is slightly broader than the proton distribution because of its larger diffusivity $\left(a_{n}-a_{p} \approx 0.1 \mathrm{fm}\right)$, but has the same half radius as the proton distribution $\left(R_{p} \approx R_{n} \approx 6.7 \mathrm{fm}\right)$. For the LEAR measurement no uncertainty was explicitly reported for $a_{n}$ though the central value is consistent with Ref. [12] and we assume $\pm 0.03 \mathrm{fm}$; both use the same proton charge density parameters taken from the ADND. The neutron parameters are then averaged and listed in Table IV together with the proton point $2 \mathrm{pF}$ parameters. The combined point density distribution for protons and neutrons is then the weighted sum of the individual $2 \mathrm{pF}$ distributions, which we simulate in the MCG by drawing 82 protons from the proton point $2 \mathrm{pF}$ and 128 neutrons from the neutron point $2 \mathrm{pF}$. The $\mathrm{D} 2 \mathrm{pF}$ distribution is displayed in Fig. 2 with its corresponding $1 \sigma$ uncertainty and compared to the traditionally used charge density distribution.

The relative change in $N_{\text {coll }}$ due to switching from the $2 \mathrm{pF}$ charge density to the $\mathrm{D} 2 \mathrm{pF}$ point density representation (while everything else is computed in the traditional approach) is

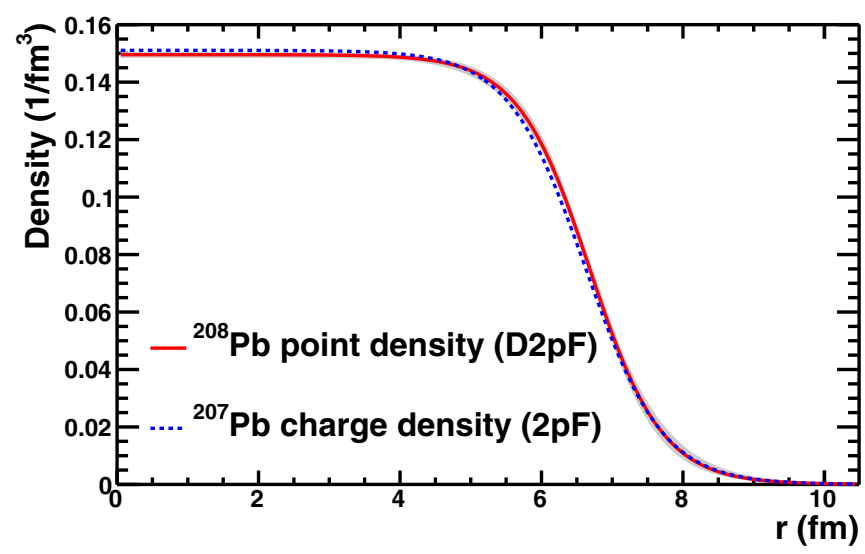

FIG. 2. Nuclear density of ${ }^{208} \mathrm{~Pb}$ for the charge distribution $(2 \mathrm{pF})$ as well as the sum of the proton and neutron point density distributions $(\mathrm{D} 2 \mathrm{pF})$, corresponding to the parameters listed in Table IV. The grey band indicates the $1 \sigma$ uncertainty for the $\mathrm{D} 2 \mathrm{pF}$ distribution.

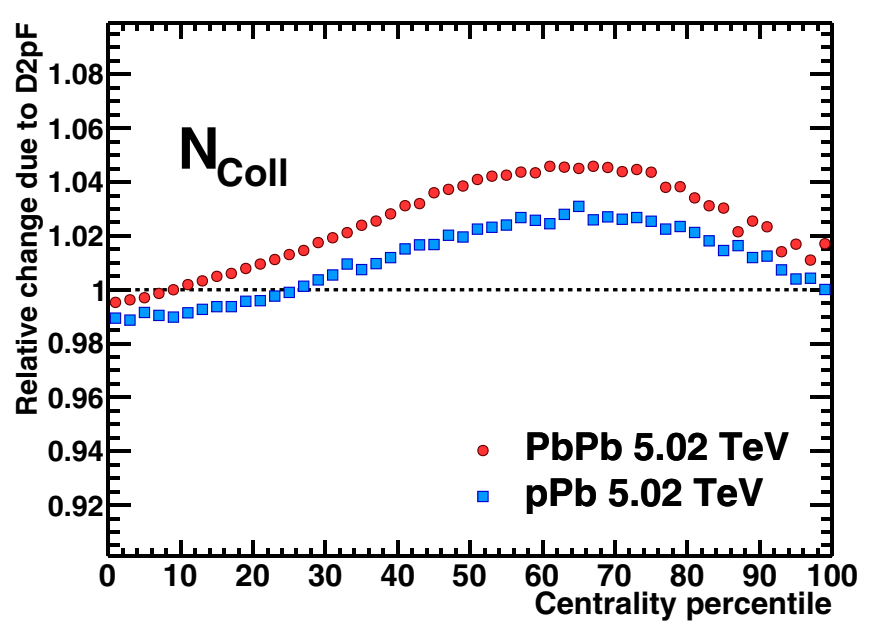

FIG. 3. Relative change in $N_{\text {coll }}$ in $\mathrm{PbPb}$ and $p \mathrm{~Pb}$ collisions at $\sqrt{s_{N N}}=5.02 \mathrm{TeV}$ due to the updated nuclear density profile. The baseline uses the standard $2 \mathrm{pF}$ charge density, while the new results are obtained with the $\mathrm{D} 2 \mathrm{pF}$ density.

illustrated in Fig. 3. In mid-peripheral $\mathrm{PbPb}$ collisions, the change results in a maximum $\sim 4 \%$ increase in $N_{\text {coll }}$ and approximately $2 \%$ for $p \mathrm{~Pb}$ collisions. This is largely driven by the increase of the central radius in the $\mathrm{D} 2 \mathrm{pF}$ compared to the $2 \mathrm{pF}$ parametrization.

\section{B. Minimum nucleon separation}

Prior to this work, varying the internucleon separation from the default value $(0.4 \mathrm{fm})$ to its assumed upper limit $(0.8 \mathrm{fm})$ led to uncertainties of about $2 \%$ in the derived Glauber quantities. Such a result is somewhat surprising given that, if uniform spherical packing is naïvely assumed for nucleons near the center of the nucleus, the typical distance between any two nucleons should be 1.5-2 fm, significantly larger than $d_{\min }$, and hence the results should not be dramatically affected when varying the latter. Traditional MCG implementations place nucleons by first sampling the $2 \mathrm{pF}$ distribution and then checking the $d_{\min }$ requirement with respect to the already placed nucleons. When the $d_{\min }$ requirement is not satisfied, the algorithm discards that nucleon and resamples the $2 \mathrm{pF}$ probability distribution. This approach results in an overall bias in the constructed radial distribution that propagates to all computed quantities. Figure 4 shows the resulting deformation in the radial profile due to this bias, which increases with increasing $d_{\min }$. Nucleons are preferentially pushed to larger radii where there is more physical phase space to fill.

One approach to overcome this effect is to rescale the input profile parameters until the bias brings the resulting density back to the desired $2 \mathrm{pF}$ distribution [59,60]. This iterative procedure is cumbersome, unphysical, and not universal for all collision systems. Instead, to remove this bias, we introduce a uniform three-dimensional lattice with a minimum nodal separation $\left(d_{\text {node }}\right)$ equivalent to $d_{\min }$. The full physical phase space is sampled by precalculating all lattice nodes within a cubic space of $40 \times 40 \times 40 \mathrm{fm}^{3}$. These nodes are sampled uniformly in Cartesian space and subsequently populated with a nucleon according to the $2 \mathrm{pF}$ probability distribution. Once a 


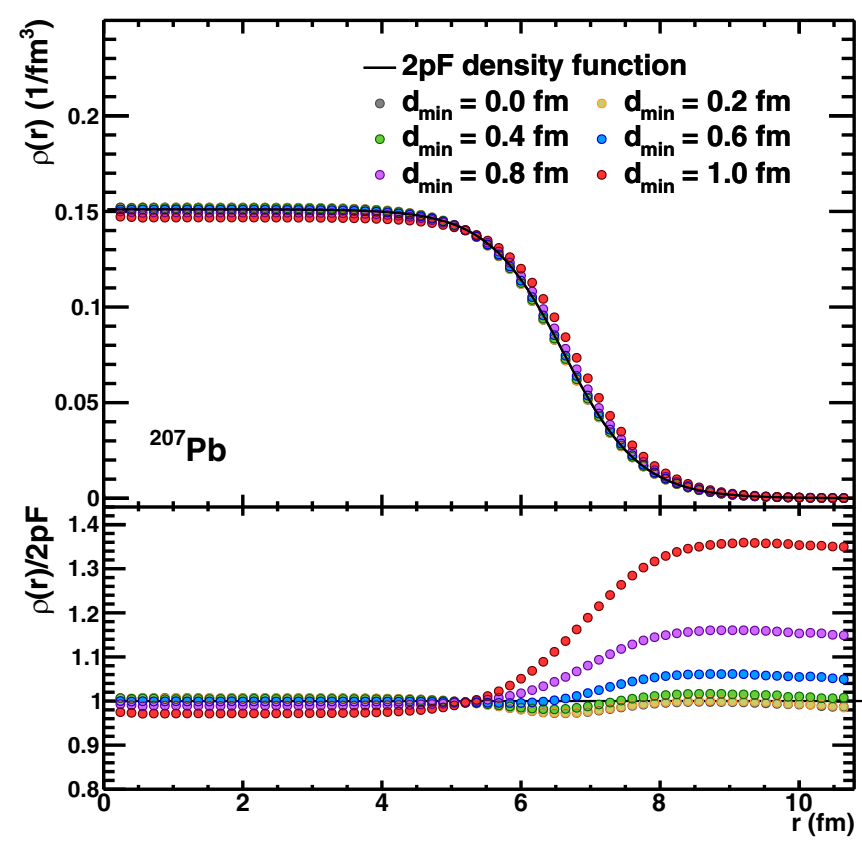

FIG. 4. Nuclear radial density distributions for various values of the internucleon distance $d_{\min }$ using the "traditional" MCG implementation (top) and their ratio to the $2 \mathrm{pF}$ profile (bottom). The deviations from 1 at large $r$ increase with increasing $d_{\min }$.

node has been populated, it is removed from the sampling. By $a$ priori restricting the allowable phase space to exclude overlapping nucleons, the $2 \mathrm{pF}$ probability distribution can be sampled without introducing artificial distortions. To ensure that regularities in the lattice are avoided, the lattice is randomized event-by-event in azimuthal and polar orientations in addition to being randomly translated laterally in Cartesian space. After the implementation of the lattice framework, the density profile remains largely intact and subsequently the centrality variables become stable with respect to $d_{\min }$ variations. This is demonstrated in Fig. 5, which shows the resulting density profiles when varying $d_{\min }$ by $100 \%(0.4 \pm 0.4 \mathrm{fm})$. The results are insensitive to the specific lattice basis used [61]: hexagonal close packed (hcp), face-centered cubic, body-centered cubic, and simple cubic. Generally, lattices with packing fractions above about $50 \%$ are indistinguishable for $d_{\min }<1.2 \mathrm{fm}$. The hcp lattice was used as the default configuration as it has the most optimal packing fraction of $74 \%$. The insensitivity to the lattice structure is intuitive when considering that less than $0.5 \%$ of nodes inside a radius of about $6.7 \mathrm{fm}$ are populated when $d_{\min }=0.4 \mathrm{fm}$. As either $d_{\min }$ is increased to larger than $1.2 \mathrm{fm}$ or the packing fraction drops significantly below $50 \%$, the fraction of nodes available will be greatly reduced and distortions start to impact the density distribution. It should be noted that, from a technical standpoint, the same result can be achieved with the traditional MCG implementation by discarding the entire nucleus in the event of two nucleons overlapping (rather than only the offending nucleon). This, however, is computationally prohibitive and therefore impractical.

Figure 6 quantifies the relative change in $N_{\text {coll }}$ with respect to the traditional MCG implementation for $d_{\min }=0.4 \mathrm{fm}$ for $\mathrm{PbPb}$ and $p \mathrm{~Pb}$ collisions. The mean value of $N_{\text {coll }}$ as a function

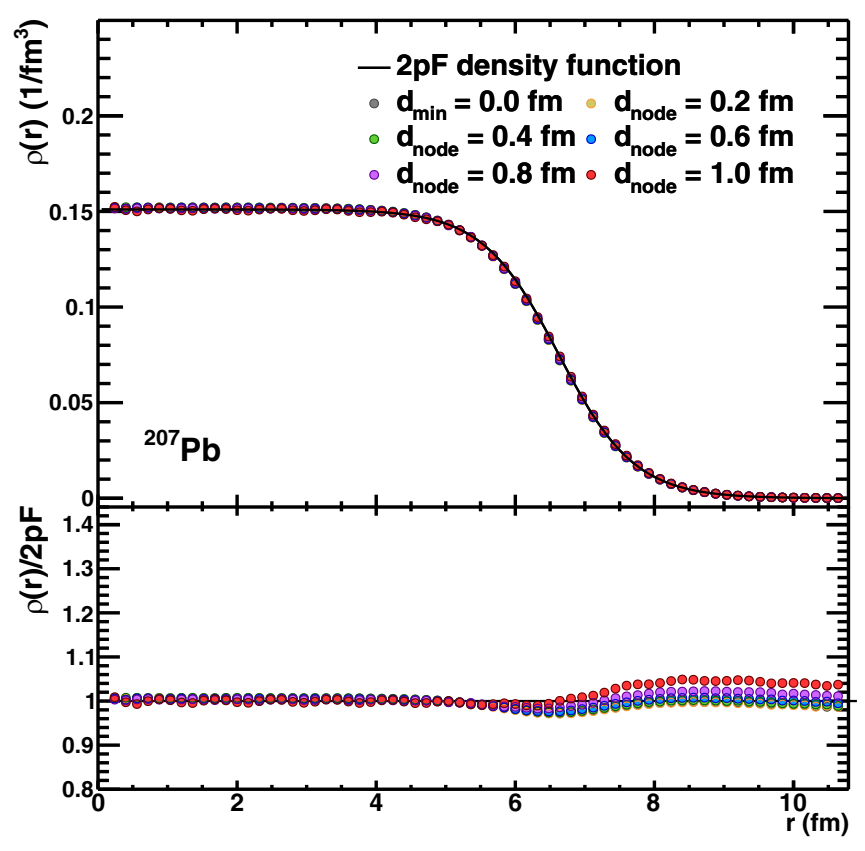

FIG. 5. Nuclear radial density distributions for various values of the internucleon lattice distance $d_{\text {node }}\left(\equiv d_{\min }\right)$ using the lattice MCG implementation (top) and their ratio to the $2 \mathrm{pF}$ profile (bottom).

of centrality changes by less than $0.2 \%$. Since the radial profile is not affected by variations of $d_{\min }$, introducing the lattice to construct the nuclei effectively removes the uncertainty due to the minimum distance between nucleons (see Sec. VI).

\section{Recentering}

Inspecting the bottom panel of Fig. 5 closely reveals that there are still residual differences of up to a few percent in the radial profile, even when the lattice is used. Indeed, even for $d_{\min }=0 \mathrm{fm}$, i.e., without a requirement on the

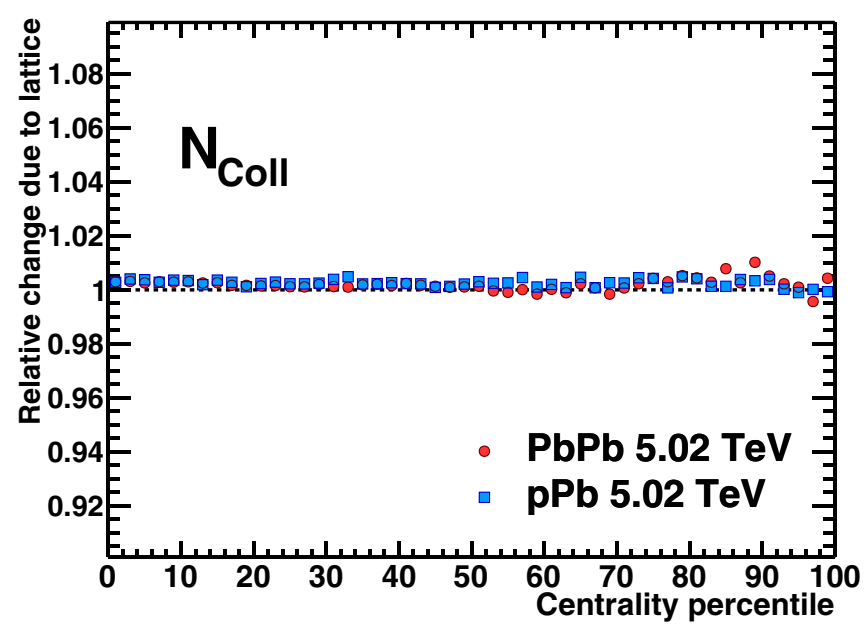

FIG. 6. Relative change in $N_{\text {coll }}$ for $\mathrm{PbPb}$ and $p \mathrm{~Pb}$ collisions at $\sqrt{s_{N N}}=5.02 \mathrm{TeV}$ after introducing the nucleon lattice placement algorithm with $d_{\text {node }}=0.4 \mathrm{fm}$. The baseline uses the traditional MCG implementation with $d_{\min }=0.4 \mathrm{fm}$. 


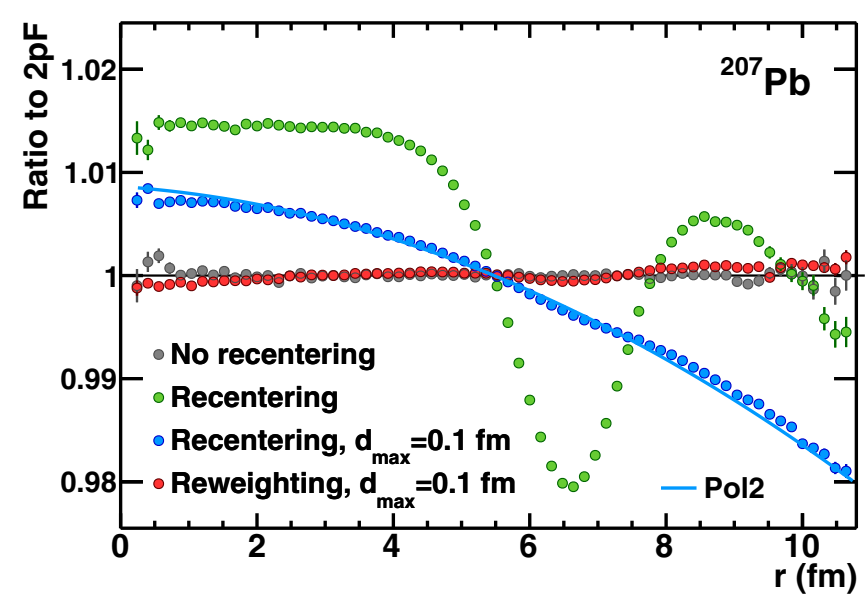

FIG. 7. Ratio of radial density distributions constructed with the standard MCG for $d_{\min }=0 \mathrm{fm}$ over that from the $2 \mathrm{pF}$ profile for different methods to recenter the nucleons. The nonmonotonous distribution is obtained with the standard approach including recentering, while the uniform distribution is without recentering. The other two cases impose $d_{\max }=0.1 \mathrm{fm}$ (as explained in the text). The mildly rising distribution is obtained by dividing (reweighting) the $\mathrm{Pb}$ nucleus radial profile with the second-order polynomial.

nucleon-nucleon separation, a nonmonotonic structure emerges, as can be seen in the "zoomed-in" ratio relative to the $2 \mathrm{pF}$ profile displayed in Fig. 7. It originates from the recentering algorithm that is usually applied in MCG calculations, since, without recentering, the ratio relative to the $2 \mathrm{pF}$ is exactly 1 . The traditional MCG approaches [6-10], except the HIJING model [5], recenter the nucleons by the average of the displacement after having distributed them individually according to the nuclear density profile. This is also the case for the advanced Monte Carlo (MC) calculation of Ref. [62], which includes realistic nucleon-nucleon correlations [63]. The recentering step is applied to ensure that the center of mass of the constructed nucleus coincides with that of the nuclear density from which the nucleon positions were stochastically determined. Shifting the nucleons by the average displacement, however, introduces a distortion of the radial profile, which increases with decreasing degrees of freedom. This effect was recently discussed in Glauber approaches accounting for subnucleonic degrees of freedom, where the distortion is particularly large when only three partons (quarks) are distributed inside a proton [64]. For a ${ }^{208} \mathrm{~Pb}$ nucleus, the width of the center-of-mass shift is about $0.2 \mathrm{fm}$ in each direction, and the effect of the associated distortions have so far been ignored. To ensure that the center of mass of the constructed nucleus is at $(0,0,0)$, one can only accept constructed nuclei where the average displacement in each direction is small, e.g., smaller than $d_{\max }=0.1 \mathrm{fm}$. This requirement leads to more (less) dense radial distributions than the $2 \mathrm{pF}$ profile for small (large) radii, as can be seen in the corresponding ratio (blue curve) in Fig. 7. The corresponding ratio can be empirically described by a second-order polynomial as $f(r)=1.00863-0.00045 r-0.00021 r^{2}$. Reweighting the original radial profile with $f$, i.e., using $\rho / f$ to distribute nucleons in the radial direction, allows to

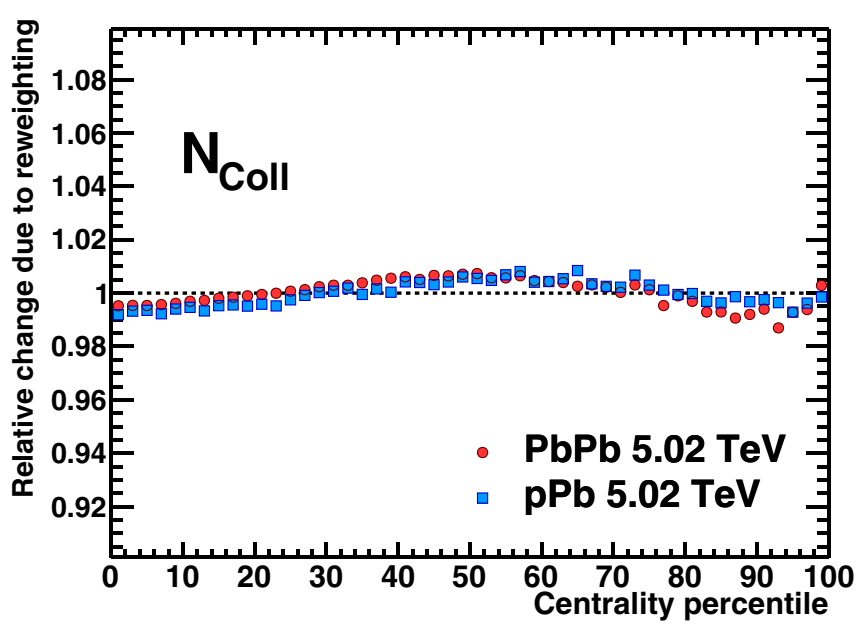

FIG. 8. Relative change in $N_{\text {coll }}$ in $\mathrm{PbPb}$ and $p \mathrm{~Pb}$ collisions at $\sqrt{s_{N N}}=5.02 \mathrm{TeV}$ using the reweighted profile for $d_{\min }=0.1 \mathrm{fm}$ and $d_{\text {min }}=0.4$. The baseline uses the traditional MCG implementation with $d_{\min }=0.4 \mathrm{fm}$.

correct for the residual bias. The ratio of the resulting radial distribution relative to the $2 \mathrm{pF}$ distribution deviates by less than $0.2 \%$ from unity.

The effect on $N_{\text {coll }}$ of the reweighted radial profile relative to the standard $2 \mathrm{pF}$ profile is quantified in Fig. 8 and leads to variations below $1 \%$. In particular, for $p \mathrm{~Pb}$ collisions the residual change from recentering to reweighting is much smaller than the modification introduced by recentering alone, which for peripheral collisions is larger than $10 \%$ (see Fig. 20).

\section{Nucleon-nucleon collision modeling}

Given that the nucleon-nucleon interaction probability depends on the condition given by Eq. (11), the improved $\sqrt{s}$ parametrization and uncertainty of $\sigma_{N N}$ discussed in Sec. III leads to Glauber quantities that are both more accurate and more precise than before.

To demonstrate the effect of this change for $\sqrt{s_{N N}}=$ $5.02 \mathrm{TeV}$, the relative change of $N_{\text {coll }}$ with respect to the previously used $\sigma_{N N}$ value is shown in Fig. 9. The baseline uses the value of $\sigma_{N N}=70 \mathrm{mb}$, commonly used at the LHC, while the updated value is $67.6 \mathrm{mb}$. As expected, the change is largest for central collisions, namely, equal to the ratio of $67.6 / 70 \approx 0.97$, while for the most peripheral collisions there is no observable numerical change.

\section{RESULTS}

The improvements considered here, including the $\sqrt{s}$ parametrization of $\sigma_{N N}$, the use of the $\mathrm{D} 2 \mathrm{pF}$ profile, plus lattice regularization, as well as the recentering and reweighting approach, comprise the improved MCG approach [17], whose parameters are summarized in Table III. To illustrate the differences with the traditional approach, we compare the values of $N_{\text {coll }}, N_{\text {part }}, T_{A A}$, and $\varepsilon_{2}$ computed with both approaches for $\mathrm{PbPb}$ and $p \mathrm{~Pb}$ collisions at $\sqrt{s_{N N}}=5.02 \mathrm{TeV}$ in Figs. 10 and 11. The uncertainties due to the $2 \mathrm{pF}$ and $\mathrm{D} 2 \mathrm{pF}$ parameters, given in Table IV, were calculated by running 


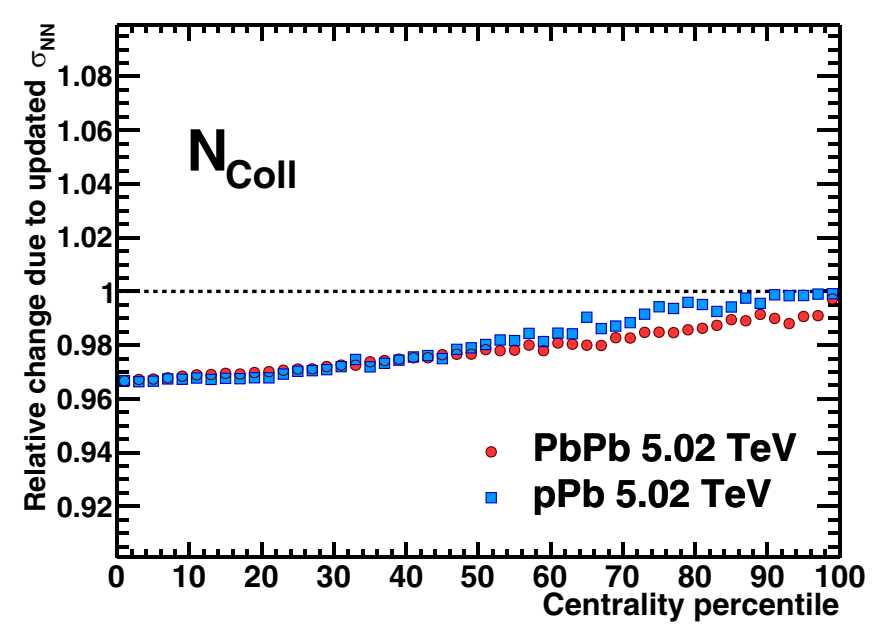

FIG. 9. Relative change in $N_{\text {coll }}$ in $\mathrm{PbPb}$ and $p \mathrm{~Pb}$ collisions at $\sqrt{s_{N N}}=5.02 \mathrm{TeV}$ due to the updated nucleon-nucleon cross section. The baseline uses $\sigma_{N N}=70 \mathrm{mb}$, while the updated value is $67.6 \mathrm{mb}$.

$1 \times 10^{5}$ MCG events for 100 parameter set variations. Each variation allowed each parameter to take a random value within a Gaussian distribution with a width of the $1 \sigma$ uncertainty on each parameter. The spread of the resulting values quantified by their standard deviation was used as the reported resulting $1 \sigma$ uncertainty due to the $2 \mathrm{pF}$ and $\mathrm{D} 2 \mathrm{pF}$ parameters. The uncertainties due to $\sigma_{N N}$ as well as due to the minimum internuclear separation $\left(d_{\min }\right.$ and $\left.d_{\text {node }}\right)$, given in Table III, were obtained by running with nominal settings, varying each
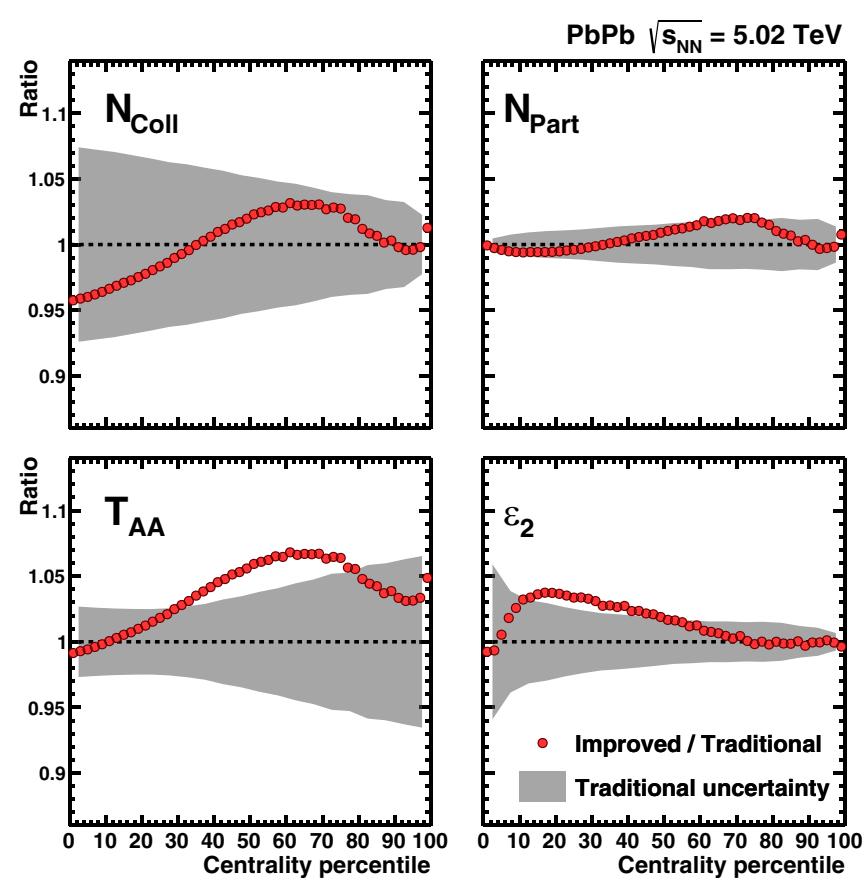

FIG. 10. Ratio of $N_{\text {coll }}, N_{\text {part }}, T_{A A}$, and $\varepsilon_{2}$ as a function of centrality obtained using the improved approach (including $\sqrt{s_{N N}}$, lattice, and $\mathrm{D} 2 \mathrm{pF}$ changes) over the same quantities obtained with the traditional approach, for $\mathrm{PbPb}$ collisions at $\sqrt{s_{N N}}=5.02 \mathrm{TeV}$. The grey band illustrates the traditionally reported uncertainties.
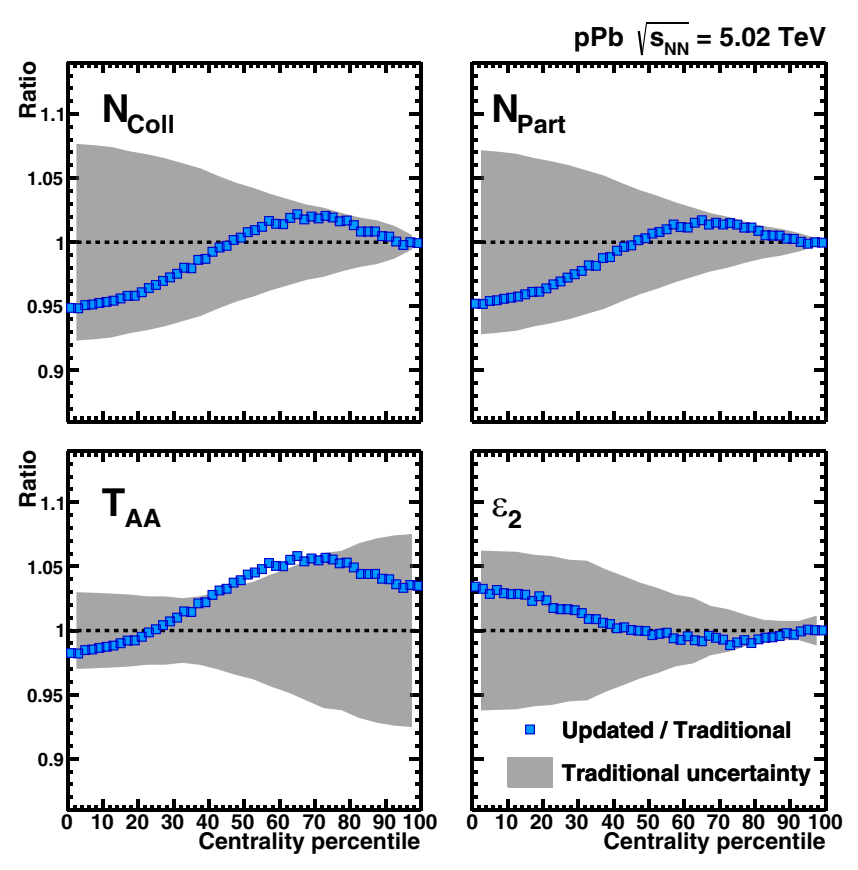

FIG. 11. Ratio of $N_{\text {coll }}, N_{\text {part }}, T_{A A}$, and $\varepsilon_{2}$ as a function of centrality obtained using the improved approach (including $\sqrt{s_{N N}}$, lattice, and $\mathrm{D} 2 \mathrm{pF}$ changes) over the same quantities obtained with the traditional approach, for $p \mathrm{~Pb}$ collisions at $\sqrt{s_{N N}}=5.02 \mathrm{TeV}$. The grey band illustrates the traditionally reported uncertainties.

one of the parameters by $\pm \sigma$ at a time, and assigning half of the difference as the corresponding $1 \sigma$ uncertainty. To obtain the total uncertainties the individual uncertainties due to density profile, $\sigma_{N N}$, and internucleon separation were added in quadrature.

Figures 10 and 11 quantify the changes as the ratio of $N_{\text {coll }}, N_{\text {part }}, T_{A A}$, and $\varepsilon_{2}$ in 5\%-wide centrality intervals using the improved and traditional approach for $\mathrm{PbPb}$ and $p \mathrm{~Pb}$ collisions at $\sqrt{s_{N N}}=5.02 \mathrm{TeV}$, respectively. The ratios are compared to the total uncertainties of the traditional approach to illustrate that the central values of the improved results are generally within the previously assigned uncertainties, which were typically dominated by the large uncertainty on $\sigma_{N N}$, except in the case of $T_{A A}$ for peripheral $\mathrm{PbPb}$ collisions. Since in $T_{A A}$ the quite large uncertainties on $\sigma_{N N}$ cancel out, this quantity is especially sensitive to other small changes introduced by the improvements. Our results indicate that, for the relevant centrality classes, previous experimental results on $R_{A A}$ would have to be scaled up by up to $3-5 \%$; however, ratios of results taking at $\sqrt{s_{N N}}=5.02$ and $\sqrt{s_{N N}}=2.76$ would not be affected because $T_{A A}$ would change similarly in both cases. We checked that the lattice and traditional approaches lead to identical results for identical settings. Hence, since it is less computationally intensive, one can also use $d_{\min }=0.4 \mathrm{fm}$ in the traditional way (i.e., without the lattice) but ignoring the uncertainties introduced from variation to $d_{\min }=0$ and $0.8 \mathrm{fm}$.

Figures 12 and 13 show the respective individual and total uncertainties as dotted lines for the traditional and solid lines for the improved model. The $2 \mathrm{pF}$ uncertainties and the minimum internucleon separation $\left(d_{\min }\right)$ reach up to about $2 \%$, while the (previously) large uncertainty on $\sigma_{N N}$ propagated into 

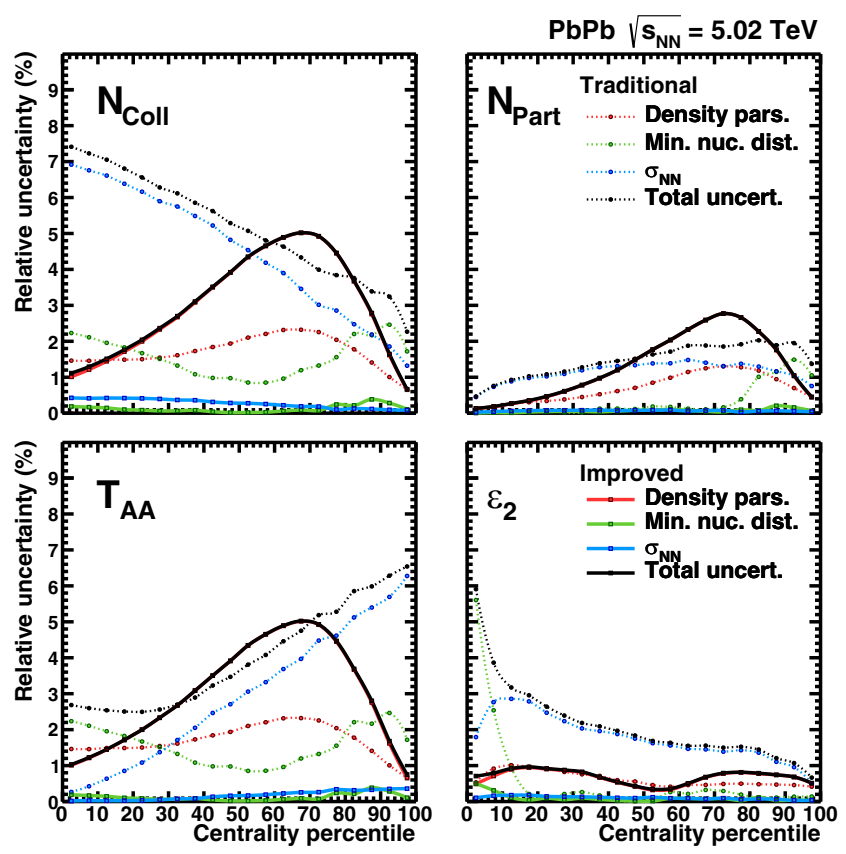

FIG. 12. Relative uncertainties in $N_{\text {coll }}, N_{\text {part }}, T_{A A}$, and $\varepsilon_{2}$ arising from varying $\sigma_{N N}$, as well as internucleon separation and density parameters for the improved and traditional approaches in $\mathrm{PbPb}$ collisions at $\sqrt{s_{N N}}=5.02 \mathrm{TeV}$.

up to $7 \%$ on $N_{\text {coll }}$ for central collisions and $T_{A A}$ for peripheral collisions, and typically dominated the final uncertainty. In contrast, the uncertainties due to the minimum separation enforced between nucleons by the lattice as well as due to the more precise parametrization of $\sigma_{N N}\left(\sqrt{s_{N N}}\right)$ are quite small and, in particular, for the internucleon separation nearly negligible in the improved approach. This is particularly apparent in the case of $\varepsilon_{2}$, where the uncertainty related to $d_{\min }$ was substantial in central collisions for the traditional approach. The uncertainty due to the $\mathrm{D} 2 \mathrm{pF}$ parameters, however, have grown. Since the uncertainty on the neutron diffusivity has actually increased to about 5\% and there are about 50\% more neutrons than protons in ${ }^{208} \mathrm{~Pb}$, the inclusion of the $\mathrm{D} 2 \mathrm{pF}$ nuclear density description results in a more accurate, though less precise, determination of Glauber quantities. For this reason, coupled with the fact that traditional $2 \mathrm{pF}$ forms represented the charge density rather than the point density, previously quoted uncertainties based on the $2 \mathrm{pF}$ parameters were slightly underestimated. For $p \mathrm{~Pb}$ collisions, other experimental uncertainties become dominant, such as those resulting from the event activity class used to determine the centrality [51]. Furthermore, it is important to realize that the spread of the computed quantities in each centrality class is rather large, in particular for peripheral collisions, where the ratios of the standard deviation over the mean of each Glauber quantity can reach up to 80\% (as can be seen in the tables of Appendix D).

Figure 14 shows the average fraction of $p p, p n$, and $n n$ collisions for $\mathrm{PbPb}$ and $p \mathrm{~Pb}$ collisions at $\sqrt{s_{N N}}=5.02 \mathrm{TeV}$ from the $\mathrm{D} 2 \mathrm{pF}$ calculation. In peripheral collisions, the $p n$ and $n n$ interactions become more probable due to the extended neutron halo or skin, and therefore are particularly relevant
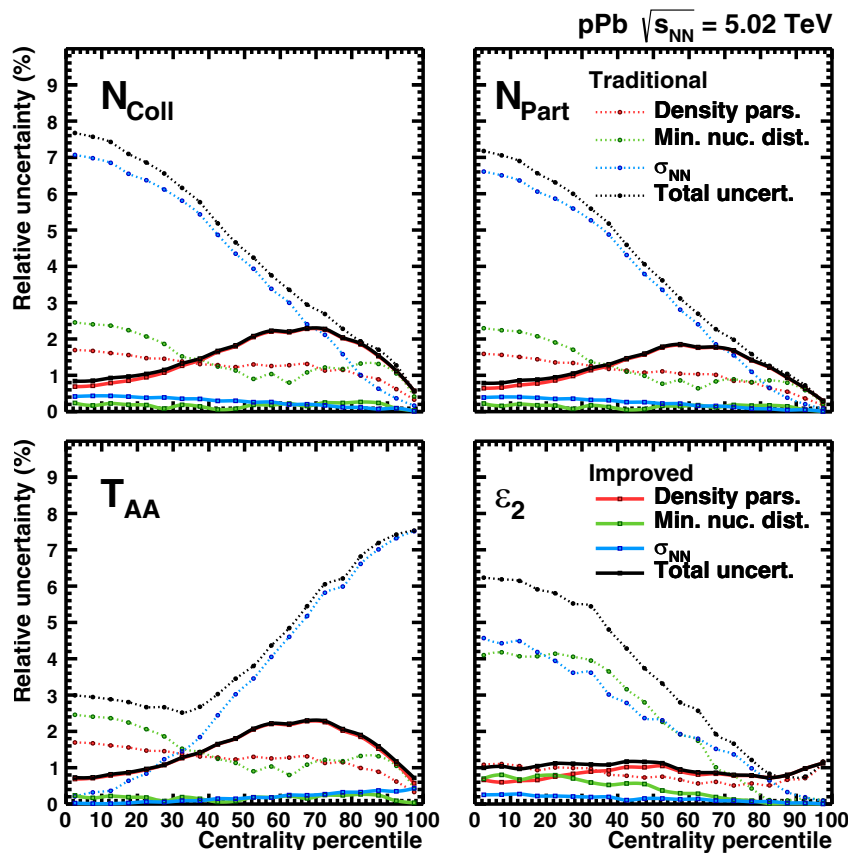

FIG. 13. Relative uncertainties in $N_{\text {coll }}, N_{\text {part }}, T_{A A}$, and $\varepsilon_{2}$ arising from varying $\sigma_{N N}$ as well as internucleon separation and density parameters for the improved and traditional approaches in $p \mathrm{~Pb}$ collisions at $\sqrt{s_{N N}}=5.02 \mathrm{TeV}$.

for precision measurements involving isospin-dependent (or electric charge-dependent) observables, such as electroweak boson production, in nuclear collisions [13-15].

Finally, we present the number of binary collisions $\left(N_{\text {coll }}\right)$, number of participants $\left(N_{\text {part }}\right)$, and overlap $\left(T_{A A}\right)$ in Fig. 15, as well as the overlap area $\left(A_{\mathrm{T}}\right)$, average path length $(L)$, participant eccentricity $\left(\varepsilon_{2}\right)$, and triangularity $\left(\varepsilon_{3}\right)$ in Fig. 16 as a function of centrality for $\mathrm{PbPb}$ collisions at $\sqrt{s_{N N}}=2.76$, 5.02 , and $39 \mathrm{TeV}$ (left plots), and in $p \mathrm{~Pb}$ collisions at $\sqrt{s_{N N}}=$ $5.02,8.16$, and $63 \mathrm{TeV}$ (right plots) using the improved MCG. The inelastic cross sections for all collision systems computed

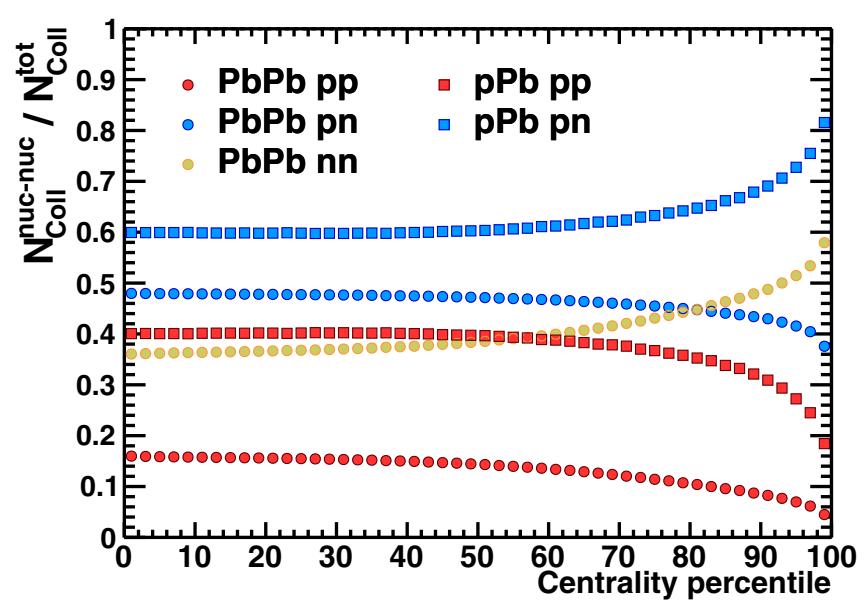

FIG. 14. Average fraction of $p p, p n$, and $n n$ collisions for $\mathrm{PbPb}$ and $p \mathrm{~Pb}$ collisions at $\sqrt{s_{N N}}=5.02 \mathrm{TeV}$ obtained with our improved MCG model. 

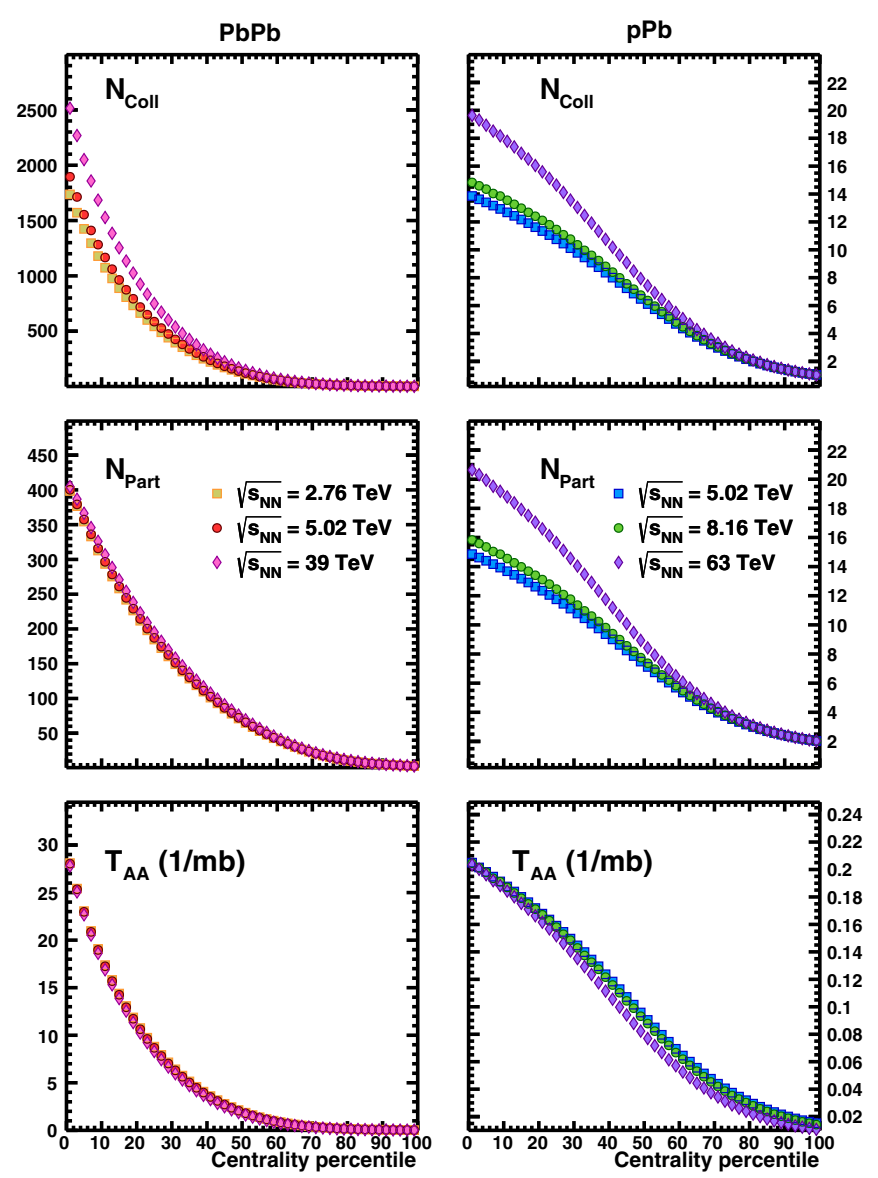

FIG. 15. Number of binary collisions $\left(N_{\text {coll }}\right)$, number of participants $\left(N_{\text {part }}\right)$, and overlap function $\left(T_{A A}\right)$ as a function of centrality for $\mathrm{PbPb}$ collisions at $\sqrt{s_{N N}}=2.76,5.02$, and $39 \mathrm{TeV}$ (left columns) and for $p \mathrm{~Pb}$ collisions at $\sqrt{s_{N N}}=5.02,8.16$, and $63 \mathrm{TeV}$ (right columns) using the improved MCG.

with the improved approach are given in Table V. Appendix D provides detailed tables for the corresponding quantities in $5 \%$-wide centrality classes.

\section{SUMMARY}

We have presented the results of an improved Monte Carlo Glauber model for the calculation of quantities of relevance for collisions involving nuclei at center-of-mass energies of BNL RHIC $\left(\sqrt{s_{N N}}=0.2 \mathrm{TeV}\right)$, CERN LHC $\left(\sqrt{s_{N N}}=\right.$ $2.76-8.8 \mathrm{TeV})$, and proposed future hadron colliders $\left(\sqrt{s_{N N}} \approx\right.$ $10-63 \mathrm{TeV})$. The corresponding values for the inelastic $p p$ cross section are obtained from a data-driven parametrization resulting in a tenfold reduction of the uncertainties due to the many available measurements at LHC collision energies (Fig. 1). We describe the nuclear transverse density with two independent two-parameter Fermi distributions for protons and neutrons to account for their different densities close to the nuclear periphery. Furthermore, we model the nucleon degrees of freedom inside a nucleus using a lattice with a minimum nodal separation to enforce the exclusion of overlapping nucleons without distorting the nuclear density. Residual small distortions in the generated nuclear densities, resulting from
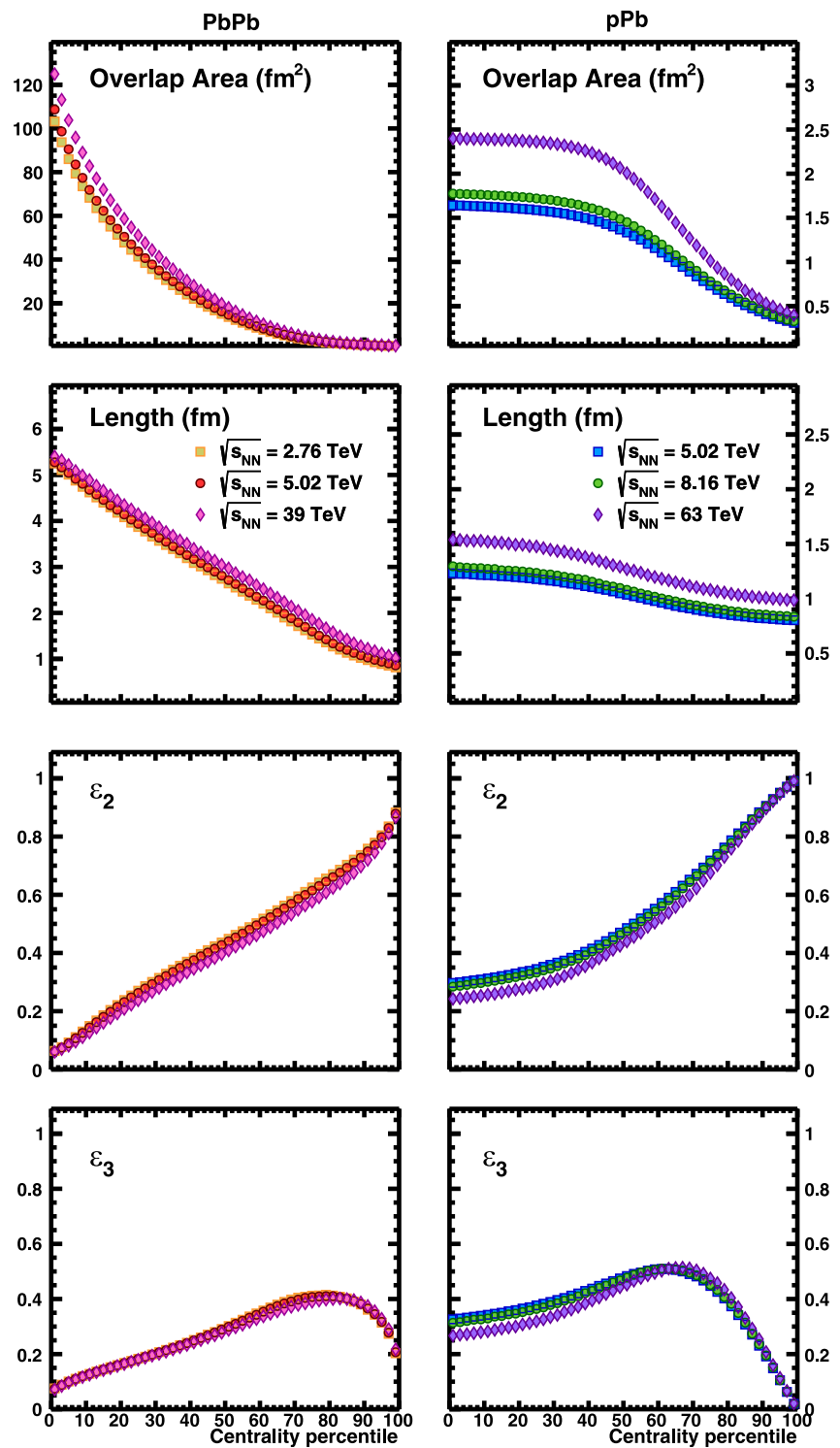

FIG. 16. Overlap area $\left(A_{\mathrm{T}}\right)$, average path length $(L)$, participant eccentricity $\left(\varepsilon_{2}\right)$, and triangularity $\left(\varepsilon_{3}\right)$ as a function of centrality for $\mathrm{PbPb}$ collisions at $\sqrt{s_{N N}}=2.76,5.02$, and $39 \mathrm{TeV}$ (left columns) and for $p \mathrm{~Pb}$ collisions at $\sqrt{s_{N N}}=5.02,8.16$, and $63 \mathrm{TeV}$ (right columns) using the improved MCG.

adjusting the nucleon center of mass with that of the nucleus, are overcome by appropriately reweighting the original nuclear density. We demonstrate for collisions at $\sqrt{s_{N N}}=5.02 \mathrm{TeV}$ that the central values of the first four quantities change due to the inclusion of the separated proton and neutron transverse distributions, though they remain typically within the previously assigned systematic uncertainties, while their new associated uncertainties are generally smaller than for earlier calculations (Figs. 10-13). The number of participant nucleons, binary nucleon-nucleon collisions, nuclear overlap function, participant eccentricity and triangularity, overlap area, and average path length are presented in intervals of percentile centrality for lead-lead $(\mathrm{PbPb})$ and proton-lead $(p \mathrm{~Pb})$ collisions at all collisions energies (Figs. 15 and 16). 
TABLE V. Values for total $\mathrm{PbPb}$ and $p \mathrm{~Pb}$ cross sections (with statistical uncertainties) at collision energies relevant for the LHC and FCC. For completeness, results for XeXe at $\sqrt{s_{N N}}=5.44 \mathrm{TeV}$ as well as for $\mathrm{AuAu}$ and $\mathrm{CuCu}$ collisions at $\sqrt{s_{N N}}=0.2 \mathrm{TeV}$ are also included. The values for $\sigma_{N N}$ are from Table II. For every collision system the corresponding centrality-dependent Glauber quantities can be found in the specified table provided in Appendix D.

\begin{tabular}{lcccc}
\hline \hline$\sqrt{s_{N N}(\mathrm{TeV})}$ & $\sigma_{N N}(\mathrm{mb})$ & System & $\sigma(\mathrm{b})$ & Table \\
\hline 2.76 & $61.8 \pm 0.9$ & $\mathrm{PbPb}$ & $7.57 \pm 0.03$ & $\mathrm{IX}$ \\
5.02 & $67.6 \pm 0.6$ & $\mathrm{PbPb}$ & $7.66 \pm 0.03$ & $\mathrm{X}$ \\
5.5 & $68.5 \pm 0.5$ & $\mathrm{PbPb}$ & $7.67 \pm 0.03$ & $\mathrm{XI}$ \\
10.6 & $75.3 \pm 0.7$ & $\mathrm{PbPb}$ & $7.77 \pm 0.03$ & $\mathrm{XII}$ \\
39 & $90.5 \pm 3.3$ & $\mathrm{PbPb}$ & $7.90 \pm 0.03$ & $\mathrm{XIII}$ \\
5.02 & $67.6 \pm 0.6$ & $p \mathrm{~Pb}$ & $2.08 \pm 0.01$ & $\mathrm{XIV}$ \\
8.16 & $72.5 \pm 0.5$ & $p \mathrm{~Pb}$ & $2.12 \pm 0.01$ & $\mathrm{XV}$ \\
8.8 & $73.3 \pm 0.6$ & $p \mathrm{~Pb}$ & $2.13 \pm 0.01$ & $\mathrm{XVI}$ \\
17 & $80.6 \pm 1.5$ & $p \mathrm{~Pb}$ & $2.18 \pm 0.01$ & $\mathrm{XVII}$ \\
63 & $96.5 \pm 4.6$ & $p \mathrm{~Pb}$ & $2.28 \pm 0.01$ & $\mathrm{XVIII}$ \\
5.44 & $68.4 \pm 0.5$ & $\mathrm{XeXe}$ & $5.67 \pm 0.02$ & $\mathrm{XIX}$ \\
0.2 & $41.6 \pm 0.6$ & $\mathrm{AuAu}$ & $6.80 \pm 0.03$ & $\mathrm{XX}$ \\
0.2 & $41.6 \pm 0.6$ & $\mathrm{CuCu}$ & $3.43 \pm 0.03$ & $\mathrm{XXI}$ \\
\hline \hline
\end{tabular}

Tables for all quantities versus centrality at present and foreseen collision energies involving $\mathrm{Pb}$-nuclei, but also for $\mathrm{XeXe}$ at $\sqrt{s_{N N}}=5.44 \mathrm{TeV}$, and for $\mathrm{AuAu}$ and $\mathrm{CuCu}$ collisions at $\sqrt{s_{N N}}=0.2 \mathrm{TeV}$, are provided (see Table V). The source code for the Monte Carlo Glauber program is made publicly available in Ref. [17]. The authors welcome comments on the code and suggestions on how to make it more useful to both experimentalists and theorists.

\section{ACKNOWLEDGMENTS}

We are grateful to Klaus Reygers for common work on a first study of the impact of separated neutron and proton densities on the Glauber quantities. We thank Dan Watts for fruitful discussions regarding Ref. [12]. J.K. and C.L. are supported by the US Department of Energy, Office of Science, Office of Nuclear Physics, under Contacts No. DE-FG02-94ER40865 and No. DE-AC05-00OR22725, respectively.

\section{APPENDIX A: COMPARISON WITH OPTICAL GLAUBER}

As described in Sec. II, the underlying Glauber formalism is the same for optical and MC calculations. Nevertheless, as discussed in Ref. [1], there are differences in their results, particularly in peripheral collisions. This is demonstrated in Fig. 17, where $N_{\text {coll }}$ in peripheral collisions deviates strongly between an optical and the Monte Carlo Glauber calculation. The optical Glauber calculation [65] was performed with the same parameters for the $2 \mathrm{pF}$ distribution of $\mathrm{Pb}$. Similarly, the proton was described in the same way, namely, with an exponential distribution $\exp (-r / R)$ with $R=0.234 \mathrm{fm}$ based on the measured form factor of the proton [66]. For peripheral $\mathrm{PbPb}$ collisions beyond $60 \%$ centrality the two calculations differ by more than $20 \%$, and in the case of $p \mathrm{~Pb}$ collisions the ratio is even nonmonotonous. Optical calculations, which

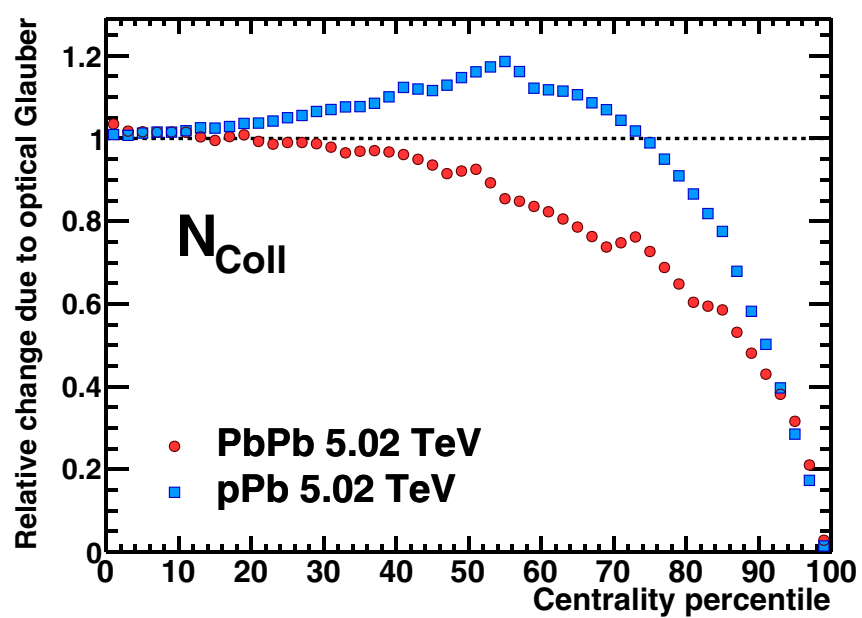

FIG. 17. Relative change in $N_{\text {coll }}$ in $\mathrm{PbPb}$ and $p \mathrm{~Pb}$ collisions at $\sqrt{s_{N N}}=5.02 \mathrm{TeV}$ due to the use of the optical Glauber model. The baseline uses the traditional MCG approach with $\sigma_{N N}=70 \mathrm{mb}$.

assume a smooth matter distribution, and by construction cannot capture event-by-event fluctuations, should not be trusted in this regime.

\section{APPENDIX B: SUBNUCLEONIC DEGREES OF FREEDOM}

Potential improvements can be added to the MCG model to take into account subnucleonic dynamics in the nuclear collision, by adding parton degrees of freedom [10], or fluctuations in the nucleon shape (also known as Glauber-Gribov fluctuations) [67]. The TGlauberMC includes the possibility that $p p$ collisions themselves have an impact parameter dependence, as, e.g., regularly taken into account in the PYTHIA event generator [68]. A convenient way to include the $b_{N N}$ dependence in MCG models is to replace the hard-sphere collision condition, $P\left(b_{N N}\right)=\Theta\left(D-b_{N N}\right)$ from Eq. (11) with

$$
P\left(b_{N N}\right)=\Gamma\left(1 / \omega, \frac{b_{N N}^{2}}{D^{2} \omega}\right) / \Gamma(1 / \omega),
$$

where $b_{N N}$ is the difference between two nucleon centers in the transverse plane, $\Gamma$ is the gamma function, and $\omega$ is a parameter which covers from the hard-sphere $(\omega=0)$ to the Gaussian $(\omega=1)$ limits.

As can be seen in Fig. 18, the resulting probability distribution approaches the hard-sphere step function for $\omega \rightarrow 0$ and a Gaussian for $\omega \rightarrow 1$. The proposed value, $\omega=0.4$, for the collisions at the LHC energies reproduces the measured values of both the total and elastic $p p$ cross sections $[9,69]$. Using $\omega=0.4$ leads to an effective reduction of the number of collisions relative to the hard-sphere condition, by about $5 \%$ and $10 \%$ in peripheral $p \mathrm{~Pb}$ and $\mathrm{PbPb}$ collisions, respectively, as shown in Fig. 19. Since the MCG calculation uses $P\left(b_{N N}\right)$ to determine whether there is a $N N$ collision, using $\omega>0$ in such calculations will impact the set of generated nucleus-nucleus collisions, and hence all Glauber quantities will change with respect to the typically applied hard-sphere $(\omega=0)$ condition, not only $N_{\text {coll }}$. The resulting change in $N_{\text {coll }}$ is qualitatively similar to earlier studies [70] on the influence of the nucleon- 


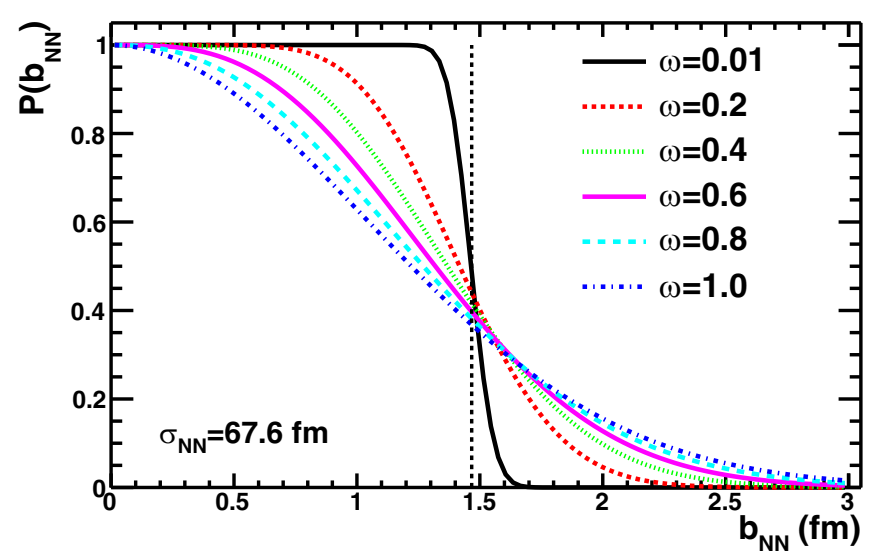

FIG. 18. Nucleon-nucleon collision impact parameter dependence $P\left(b_{N N}\right)$ from Eq. (B1) for various values of $\omega$, at $5.02 \mathrm{TeV}$. The vertical dashed line $(\omega=0)$ corresponds to the hard-sphere limit.

nucleon collision geometry on the determination of the $R_{A A}$. However, a realistic modeling of the number of hard collisions per $N N$ collision, and in general of the correlation between soft and hard particle production, is needed to be able to compare experimental data with calculations [71].

\section{APPENDIX C: USER'S GUIDE}

The source code, which relies on the ROOT [72] framework (version 4.00/08 or higher), can be obtained at the TGlauberMC page on HepForge [17]. All functionality is implemented in the macro runglauber_vX.Y.C, where version "X.Y $\mathrm{Y}=3.0 "$ described here. For generating events with ${ }^{3} \mathrm{H}$ or ${ }^{3} \mathrm{He}$, the additional text files called "h3_plaintext.dat" or "he3_plaintext.dat" are needed. Compiling the code is done as in earlier versions, namely, by executing

root [0] gSystem->Load ("libMathMore")

root [1].L runglauber_3.0.C+

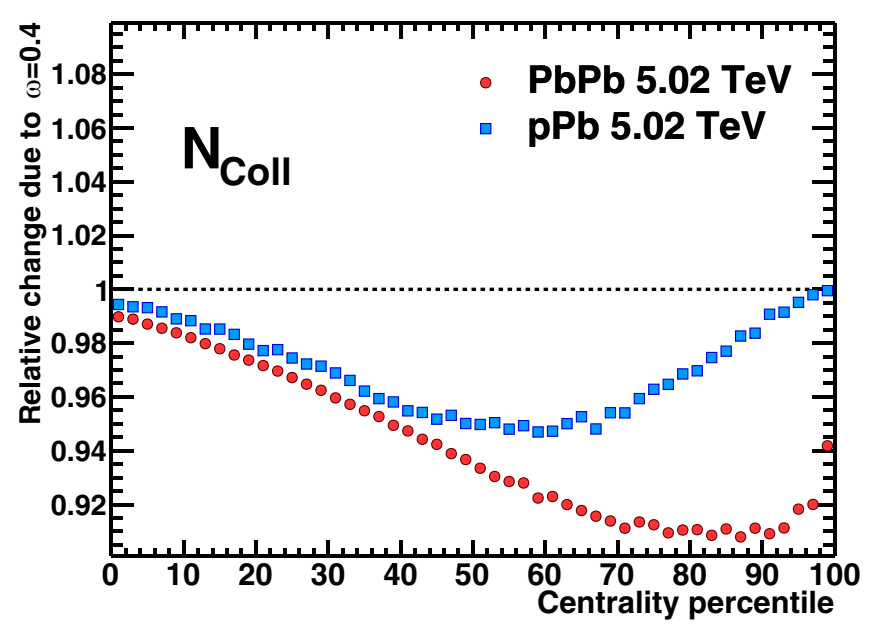

FIG. 19. Relative change in $N_{\text {coll }}$ in $\mathrm{PbPb}$ and $p \mathrm{~Pb}$ collisions at $\sqrt{s_{N N}}=5.02 \mathrm{TeV}$ due to the use of $\omega=0.4 \mathrm{in} \mathrm{Eq.} \mathrm{(B1)} \mathrm{instead} \mathrm{of}$ the hard-sphere condition.
TABLE VI. Nuclear density parameters for charge density distributions of $\mathrm{Cu}, \mathrm{Xe}$, and $\mathrm{Au}$ (see Ref. [7]). The name of the corresponding profile in the TGlauberMC implementation is also listed. See Table IV for $\mathrm{Pb}$. Separate proton and neutron point densities are not known. The values for xenon are obtained from $R=5.4 \pm 0.1 \mathrm{fm}$ and $a=0.61_{-0.09}^{+0.07} \mathrm{fm}$ for ${ }^{132} \mathrm{Xe}$ from Ref. [73], where the radius was scaled down by $0.99=(129 / 132)^{1 / 3}$ and $a$ was reduced by $0.02 \mathrm{fm}$ to symmetrize the uncertainty and to approximate the smaller neutron skin of ${ }^{129} \mathrm{Xe}$.

\begin{tabular}{lccc}
\hline \hline Nucleus & Name & $R(\mathrm{fm})$ & $a(\mathrm{fm})$ \\
\hline${ }^{63} \mathrm{Cu}$ & $\mathrm{Cu}$ & $4.20 \pm 0.02$ & $0.596 \pm 0.008$ \\
${ }^{129} \mathrm{Xe}$ & $\mathrm{Xe}$ & $5.36 \pm 0.10$ & $0.590 \pm 0.070$ \\
${ }^{197} \mathrm{Au}$ & $\mathrm{Au}$ & $6.38 \pm 0.06$ & $0.535 \pm 0.027$ \\
\hline \hline
\end{tabular}

Three classes, TGlauNucleon, TGlauNucleus, and TGlauberMC, and three functions (macros), runAndSaveNtuple(), runAndSaveNucleons(), and runAndSmearNtuple (), are defined in the provided macro. In the following, we only describe the improved functionality (see Ref. [7] for the complete guide).

Executing the program can be steered by the provided runAndSaveNtuple () macro that takes the following arguments:

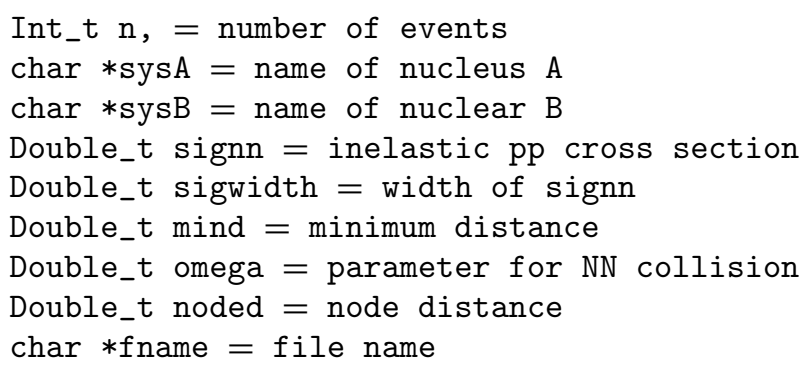

The macro will generate $n$ many Monte Carlo events and store event-by-event computed quantities in a ROOT tree, further described below, saved on disk for a given file name. If no argument for the file name is given, the code will provide it based on values given for the other arguments. The names for various nuclear profiles are listed in Tables IV and VI, and for the corresponding reweighted profiles in Tables VII and VIII. A complete list for other nuclei can be found in Ref. [7]. All implemented cases can also be found in the

TABLE VII. Values for the coefficients of the second-order polynomial used to correct the radial nuclear density distribution to cancel the effects of the recentering. The name of the corresponding profile in the TGlauberMC implementation is also listed. In case one of the reweighted parametrizations is chosen, the code will by default only generate events with $d_{\max }<0.1 \mathrm{fm}$.

\begin{tabular}{lcccc}
\hline \hline Nucleus & Name & $p_{0}$ & $p_{1} 10^{4}$ & $p_{2} 10^{4}$ \\
\hline${ }^{63} \mathrm{Cu}$ & Curw & 1.0090 & -7.9040 & -3.8990 \\
${ }^{129} \mathrm{Xe}$ & Xerw & 1.0091 & -7.2230 & -2.6630 \\
${ }^{197} \mathrm{Au}$ & Aurw & 1.0090 & -5.9091 & -2.1050 \\
${ }^{207} \mathrm{~Pb}$ & Pbrw & 1.0086 & -4.4808 & -2.0587 \\
${ }^{208} \mathrm{~Pb}$ & Pbpnrw & 1.0087 & -4.6148 & -2.0357 \\
\hline \hline
\end{tabular}


TABLE VIII. Same as Table VII for deformed nuclear profiles corresponding to density parameters given in Table VI.

\begin{tabular}{lccccc}
\hline \hline Name & $\beta_{2}$ & $\beta_{4}$ & $p_{0}$ & $p_{1} 10^{3}$ & $p_{2} 10^{5}$ \\
\hline Cu2rw & 0.162 & -0.006 & 1.0127 & -2.9808 & -9.9722 \\
Xe2arw & 0.18 & 0 & 1.0125 & -2.4851 & -5.7246 \\
Au2rw & -0.131 & -0.031 & 1.0126 & -2.2552 & -3.7151 \\
\hline \hline
\end{tabular}

TGlauNucleus : : Lookup function in the code. The value for $\sigma_{N N}$ is given in millibarns, and a variety of values for highenergy collisions can be found in Table II. In case a positive value for the width of $\sigma_{N N}$ is given, then Glauber-Gribov fluctuations (useful for $p A$ collision studies) will be simulated. As a default, a minimum separation distance of $d_{\min }=0.4 \mathrm{fm}$ is recommended. If a positive value for the node distance is given, then the nucleons will be placed on a lattice (hcp, if not otherwise specified). For values below $1 \mathrm{fm}$ the results do not depend on the node distance, but is is recommended to use $0.4 \mathrm{fm}$ for consistency with $d_{\min }$. By default no lattice will be used, and the calculation will be identical to version 2 of the code. If a positive value of $\omega$ is given, as per Eq. (B1), the determination of the number of $N N$ collisions will use an $N N$-dependent impact parameter distribution as shown in Fig. 18. Otherwise, by default, the hard-sphere condition is used.

In addition to quantities described in Ref. [7], the following quantities are stored in the ROOT tree:

Nhard: Number of hard collisions (based on fHardFrac)
Ncollpp: Number of $p p$ collisions
Ncollpn: Number of $p n$ collisions
Ncollnn: Number of $n n$ collisions
AreaW: Area defined by width of participants
Area0: Area by "or" of participants in grid
AreaA: Area by "and" of participants in grid
X0: Production point in x
Y0: Production point in y
Phi0: Direction in $\phi$
Length: Length in $\phi_{0}$

The following set of functions controls additional behavior of the TGlauberMC class: SetHardFrac(Double_t) sets the fraction of cross section used for the calculation of hard collisions (by default 0.65). SetCalcArea(Bool_t) and SetCalcLength(Bool_t) enable the calculation of the overlap area using a fine grid and the length [starting from a randomly chosen binary collision with $\left(x_{0}, y_{0}\right)$ in a random direction $\phi_{0}$ of the transverse plane]. They are by default not computed since the calculation is rather slow. SetRecenter (Int_t) specifies if and how to recenter nucleons in a nucleus, where 0 means no recentering, 1 (default) means recentering by shifting all nucleons by the average displacement, 2 means recentering by shifting only one nucleon, and 3 recentering by shifting only along the

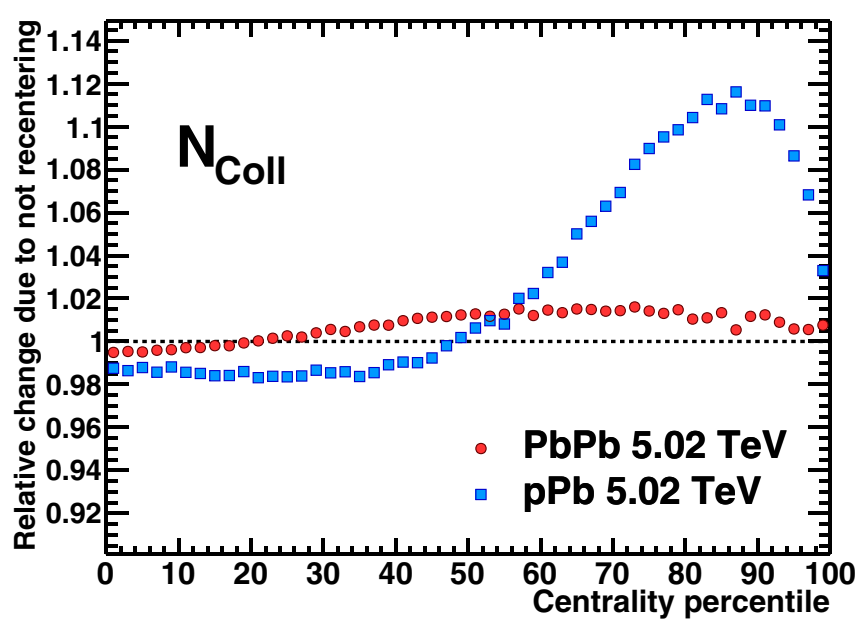

FIG. 20. Relative change in $N_{\text {coll }}$ in $\mathrm{PbPb}$ and $p \mathrm{~Pb}$ collisions at $\sqrt{s_{N N}}=5.02 \mathrm{TeV}$ for $d_{\min }=0.4 \mathrm{fm}$ without recentering compared to recentering, using the traditional MCG implementation with $d_{\min }=0.4 \mathrm{fm}$.

$z$ direction after rotating the nucleus to align the $x$ and $y$ coordinates of its center with 0 . Figure 20 demonstrates the relative change of $N_{\text {coll }}$ in $\mathrm{PbPb}$ and $p \mathrm{~Pb}$ collisions at $\sqrt{s_{N N}}=5.02 \mathrm{TeV}$ for $d_{\min }=0.4 \mathrm{fm}$ without recentering compared to recentering using the traditional MCG implementation with $d_{\min }=0.4 \mathrm{fm}$. SetShiftMax (Double_t) specifies the maximum displacement $\left(d_{\max }\right)$ of the nucleon center of mass in every direction from zero (by default any shift is accepted). SetLattice (Int_t) specifies the lattice type to use (hcp by default), and SetSmearing (Double_t) specifies the width of a Gaussian by which the nucleon position will be smeared around the lattice node position (by default not done). SetBMin (Double_t) and SetBMax (Double_t) can be used to restrict the impact parameter (by default between 0 and $20 \mathrm{fm}$ ). SetDetail (Int_t) allows one to restrict the number of variables written to the ROOT tree (by default everything is written). SetMinDistance (Double_t) defines the minimum separation distance (by default $0.4 \mathrm{fm}$ ). SetNodeDistance(Double_t) sets the node separation in the lattice mode. This value should be as large as $d_{\min }$. By default it is negative; i.e., the lattice mode is not enabled. Using SetNNProf (TF1 *) one can set another profile than that defined by Eq. (B1). See the code for how it is done for getNNProf.

\section{APPENDIX D: TABLES FOR ALL COMPUTED MCG QUANTITIES IN 5\%-WIDE CENTRALITY CLASSES}

In the following, we present the results for $N_{\text {coll }}, N_{\text {part }}, T_{A A}$, $\varepsilon_{2}, \varepsilon_{3}, A_{\mathrm{T}}$, and $L$ in $5 \%$-wide centrality classes for all systems, summarized in Table V. The centrality classes are defined by slicing the impact parameter $(b)$ distribution. For all systems at least $5 \times 10^{6}$ events were computed. For each quantity, the average and the standard deviation (labeled as rms) of the quantity in the centrality class are reported. The settings for the improved MCG model are given in Table III. 
TABLE IX. Various quantities for PbPb collisions at $\sqrt{s_{N N}}=2.76 \mathrm{TeV}$ obtained with the improved MCG model for centrality classes defined by slicing the impact parameter $(b)$ distribution. The mean and standard deviation of each quantity (denoted as rms) are given. Typical uncertainties, due to the MCG model parameters for each quantity at any centrality bin, can be read off from Fig. 12.

\begin{tabular}{|c|c|c|c|c|c|c|c|c|c|}
\hline Centrality & $b_{\min }(\mathrm{fm})$ & $b_{\max }(\mathrm{fm})$ & $\left\langle N_{\mathrm{coll}}\right\rangle \pm \mathrm{rms}$ & $\left\langle N_{\text {part }}\right\rangle \pm \mathrm{rms}$ & $\left\langle T_{A A}\right\rangle \pm \mathrm{rms}\left(\mathrm{mb}^{-1}\right)$ & $\varepsilon_{2} \pm \mathrm{rms}$ & $\varepsilon_{3} \pm \mathrm{rms}$ & $L \pm \mathrm{rms}(\mathrm{fm})$ & $A_{\perp} \pm \mathrm{rms}\left(\mathrm{fm}^{2}\right)$ \\
\hline $0-5 \%$ & 0 & 3.47 & $1615 \pm 135$ & $382 \pm 17.1$ & $26.1 \pm 2.2$ & $0.0737 \pm 0.0401$ & $0.0853 \pm 0.0455$ & $5.15 \pm 3$ & $96.3 \pm 7.44$ \\
\hline $5-10 \%$ & 3.47 & 4.91 & $1268 \pm 104$ & $328.1 \pm 17.6$ & $20.5 \pm 1.7$ & $0.116 \pm 0.059$ & $0.113 \pm 0.059$ & $4.85 \pm 2.85$ & $78 \pm 5.54$ \\
\hline $15-20 \%$ & 6.01 & 6.94 & $790.1 \pm 74.4$ & $238.2 \pm 15.8$ & $12.8 \pm 1.2$ & $0.208 \pm 0.087$ & $0.154 \pm 0.0802$ & $4.26 \pm 2.57$ & $54.2 \pm 4.29$ \\
\hline $20-25 \%$ & 6.94 & 7.76 & $618.1 \pm 66$ & $201.5 \pm 15.1$ & $10 \pm 1.1$ & $0.25 \pm 0.0986$ & $0.173 \pm 0.0899$ & $4 \pm 2.45$ & $45.3 \pm 3.99$ \\
\hline $25-30 \%$ & 7.76 & 8.5 & $478.9 \pm 59.1$ & $169.4 \pm 14.4$ & $7.75 \pm 0.96$ & $0.29 \pm 0.11$ & $0.193 \pm 0.0993$ & $3.74 \pm 2.33$ & $37.8 \pm 3.74$ \\
\hline $35-40 \%$ & 9.18 & 9.81 & $275.5 \pm 46.7$ & $116.8 \pm 13.4$ & $4.46 \pm 0.76$ & $0.36 \pm 0.132$ & $0.235 \pm 0.12$ & $3.27 \pm 2.1$ & $25.6 \pm 3.36$ \\
\hline $40-45 \%$ & 9.81 & 10.4 & $203.1 \pm 40.5$ & $95.12 \pm 12.9$ & $3.29 \pm 0.65$ & $0.393 \pm 0.143$ & $0.258 \pm 0.13$ & $3.03 \pm 1.99$ & $20.6 \pm 3.18$ \\
\hline $45-50 \%$ & 10.4 & 11 & $146.6 \pm 34.3$ & $76.37 \pm 12.2$ & $2.37 \pm 0.56$ & $0.426 \pm 0.155$ & $0.283 \pm 0.14$ & $2.81 \pm 1.89$ & $16.4 \pm 2.99$ \\
\hline $50-55 \%$ & 11 & 11.5 & $103.4 \pm 28.6$ & $60.3 \pm 11.5$ & $1.67 \pm 0.46$ & $0.458 \pm 0.168$ & $0.31 \pm 0.151$ & $2.58 \pm 1.78$ & $12.7 \pm 2.79$ \\
\hline $55-60 \%$ & 11.5 & 12 & $70.98 \pm 22.9$ & $46.59 \pm 10.7$ & $1.15 \pm 0.37$ & $0.492 \pm 0.179$ & $0.339 \pm 0.162$ & $2.35 \pm 1.68$ & $9.63 \pm 2.55$ \\
\hline $60-65 \%$ & 12 & 12.5 & $47.54 \pm 18.2$ & $35.15 \pm 9.8$ & $0.769 \pm 0.29$ & $0.529 \pm 0.191$ & $0.366 \pm 0.171$ & $2.12 \pm 1.56$ & $7.1 \pm 2.3$ \\
\hline $85-90 \%$ & 14.4 & 14.9 & $4.958 \pm 3.94$ & $6.224 \pm 3.9$ & $0.0802 \pm 0.064$ & $0.724 \pm 0.243$ & $0.388 \pm 0.278$ & $1.06 \pm 0.756$ & $1.07 \pm 0.869$ \\
\hline $90-95 \%$ & 14.9 & 15.6 & $3.206 \pm 2.68$ & $4.405 \pm 2.8$ & $0.0519 \pm 0.043$ & $0.775 \pm 0.25$ & $0.346 \pm 0.312$ & $0.94 \pm 0.579$ & $0.729 \pm 0.658$ \\
\hline $95-100 \%$ & 15.6 & 20 & $2.007 \pm 1.67$ & $3.078 \pm 1.76$ & $0.0325 \pm 0.027$ & $0.849 \pm 0.238$ & $0.252 \pm 0.326$ & $0.844 \pm 0.408$ & $0.486 \pm 0.48$ \\
\hline $0-100 \%$ & 0 & 20 & $353.3 \pm 464$ & $112.5 \pm 115$ & $5.72 \pm 7.5$ & $0.447 \pm 0.278$ & $0.273 \pm 0.206$ & $2.76 \pm 2.35$ & $25.7 \pm 28$ \\
\hline
\end{tabular}

TABLE X. Various quantities for PbPb collisions at $\sqrt{s_{N N}}=5.02 \mathrm{TeV}$ obtained with the improved MCG model for centrality classes defined by slicing the impact parameter $(b)$ distribution. The mean and standard deviation of each quantity (denoted as rms) are given. Typical uncertainties, due to the MCG model parameters for each quantity at any centrality bin, can be read off from Fig. 12.

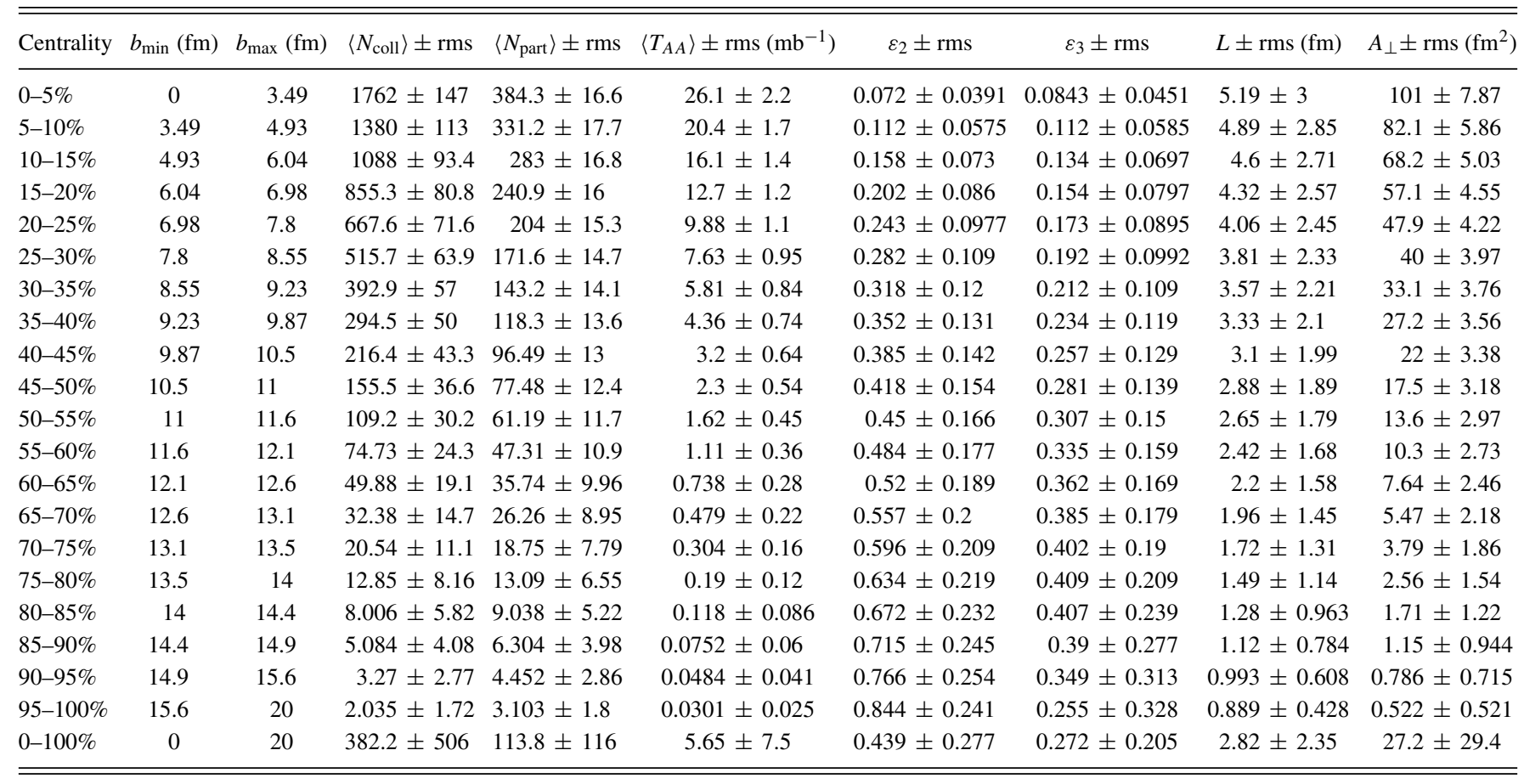


TABLE XI. Various quantities for PbPb collisions at $\sqrt{s_{N N}}=5.5 \mathrm{TeV}$ obtained with the improved MCG model for centrality classes defined by slicing the impact parameter $(b)$ distribution. The mean and standard deviation of each quantity (denoted as rms) are given. Typical uncertainties, due to the MCG model parameters for each quantity at any centrality bin, can be read off from Fig. 12.

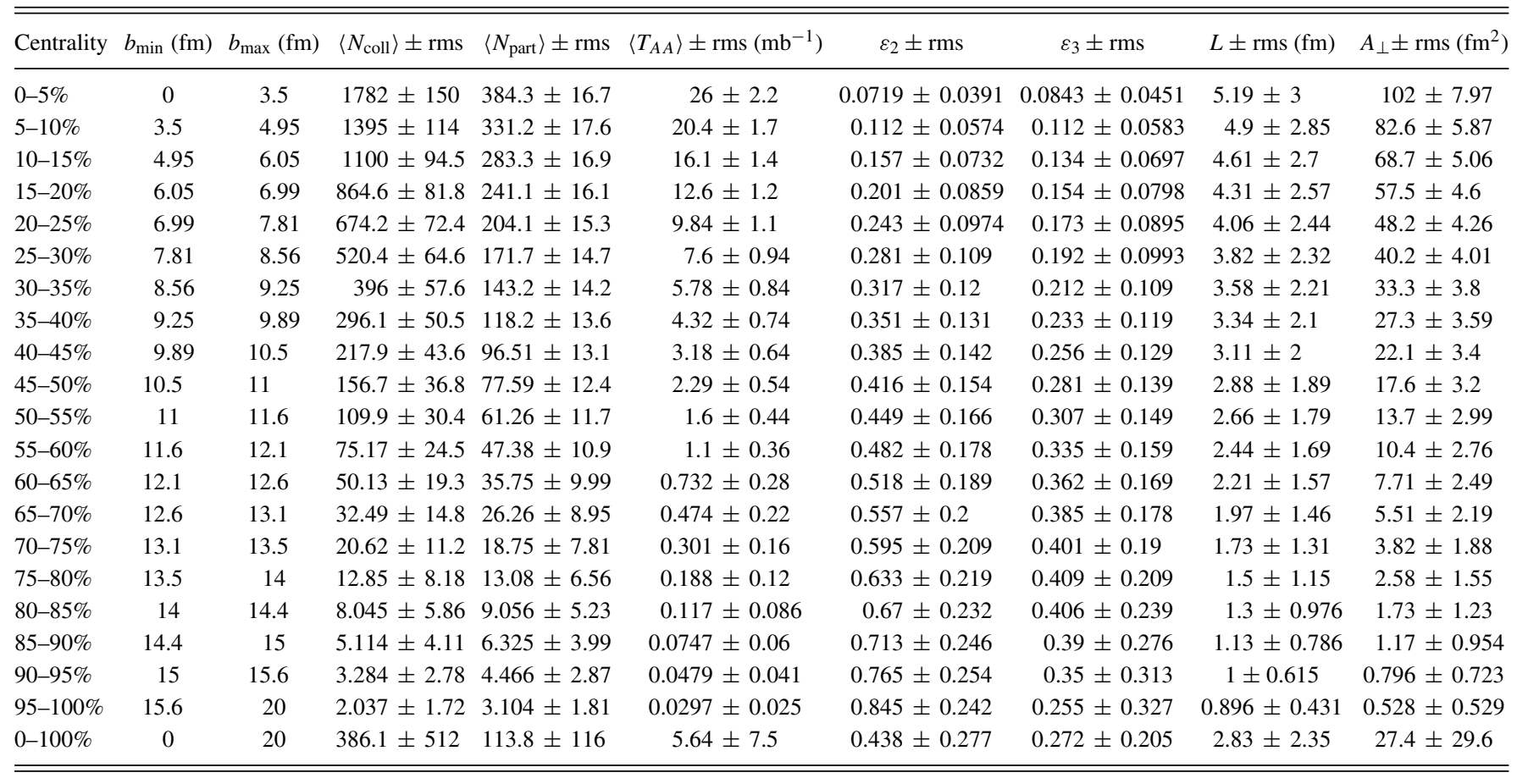

TABLE XII. Various quantities for PbPb collisions at $\sqrt{s_{N N}}=10.6 \mathrm{TeV}$ obtained with the improved MCG model for centrality classes defined by slicing the impact parameter $(b)$ distribution. The mean and standard deviation of each quantity (denoted as rms) are given. Typical uncertainties, due to the MCG model parameters for each quantity at any centrality bin, can be read off from Fig. 12.

\begin{tabular}{|c|c|c|c|c|c|c|c|c|c|}
\hline Centrality & $b_{\min }(\mathrm{fm})$ & $b_{\max }(\mathrm{fm})$ & $\left\langle N_{\text {coll }}\right\rangle \pm \mathrm{rms}$ & $\left\langle N_{\text {part }}\right\rangle \pm \mathrm{rms}$ & $\left\langle T_{A A}\right\rangle \pm \mathrm{rms}\left(\mathrm{mb}^{-1}\right)$ & $\varepsilon_{2} \pm \mathrm{rms}$ & $\varepsilon_{3} \pm \mathrm{rms}$ & $L \pm \mathrm{rms}(\mathrm{fm})$ & $A_{\perp} \pm \mathrm{rms}\left(\mathrm{fm}^{2}\right)$ \\
\hline $0-5 \%$ & 0 & 3.51 & $1954 \pm 163$ & $386.7 \pm 16$ & $26 \pm 2.2$ & $0.0703 \pm 0.0381$ & $0.0832 \pm 0.0445$ & $5.24 \pm 2.99$ & $107 \pm 8.36$ \\
\hline $5-10 \%$ & 3.51 & 4.97 & $1528 \pm 125$ & $334.8 \pm 17.5$ & $20.3 \pm 1.7$ & $0.107 \pm 0.0556$ & $0.111 \pm 0.0581$ & $4.96 \pm 2.84$ & $86.8 \pm 6.2$ \\
\hline $15-20 \%$ & 6.08 & 7.02 & $943.1 \pm 89$ & $244.5 \pm 16.2$ & $12.5 \pm 1.2$ & $0.194 \pm 0.0843$ & $0.153 \pm 0.0795$ & $4.4 \pm 2.57$ & $60.7 \pm 4.86$ \\
\hline $20-25 \%$ & 7.02 & 7.85 & $734.5 \pm 78.8$ & $207.4 \pm 15.6$ & $9.75 \pm 1$ & $0.234 \pm 0.0962$ & $0.172 \pm 0.0889$ & $4.13 \pm 2.45$ & $50.9 \pm 4.51$ \\
\hline $25-30 \%$ & 7.85 & 8.6 & $565.5 \pm 70.4$ & $174.7 \pm 14.9$ & $7.51 \pm 0.93$ & $0.272 \pm 0.107$ & $0.191 \pm 0.0986$ & $3.88 \pm 2.32$ & $42.6 \pm 4.24$ \\
\hline $35-40 \%$ & 9.29 & 9.93 & $320.2 \pm 54.8$ & $120.5 \pm 13.9$ & $4.25 \pm 0.73$ & $0.342 \pm 0.129$ & $0.232 \pm 0.118$ & $3.41 \pm 2.11$ & $29.1 \pm 3.81$ \\
\hline $40-45 \%$ & 9.93 & 10.5 & $234.2 \pm 47.1$ & $98.36 \pm 13.3$ & $3.11 \pm 0.63$ & $0.375 \pm 0.14$ & $0.254 \pm 0.128$ & $3.19 \pm 2$ & $23.6 \pm 3.62$ \\
\hline $45-50 \%$ & 10.5 & 11.1 & $167.6 \pm 39.6$ & $79.08 \pm 12.7$ & $2.23 \pm 0.53$ & $0.407 \pm 0.152$ & $0.278 \pm 0.138$ & $2.96 \pm 1.9$ & $18.9 \pm 3.42$ \\
\hline $50-55 \%$ & 11.1 & 11.6 & $117 \pm 32.5$ & $62.42 \pm 12$ & $1.55 \pm 0.43$ & $0.439 \pm 0.164$ & $0.303 \pm 0.147$ & $2.74 \pm 1.8$ & $14.7 \pm 3.2$ \\
\hline $55-60 \%$ & 11.6 & 12.2 & $79.64 \pm 26$ & $48.3 \pm 11.1$ & $1.06 \pm 0.35$ & $0.473 \pm 0.176$ & $0.329 \pm 0.157$ & $2.52 \pm 1.69$ & $11.2 \pm 2.95$ \\
\hline $60-65 \%$ & 12.2 & 12.7 & $52.8 \pm 20.4$ & $36.49 \pm 10.2$ & $0.701 \pm 0.27$ & $0.508 \pm 0.188$ & $0.355 \pm 0.166$ & $2.28 \pm 1.58$ & $8.32 \pm 2.67$ \\
\hline $85-90 \%$ & 14.5 & 15 & $5.26 \pm 4.28$ & $6.423 \pm 4.09$ & $0.0698 \pm 0.057$ & $0.701 \pm 0.248$ & $0.392 \pm 0.276$ & $1.2 \pm 0.823$ & $1.26 \pm 1.04$ \\
\hline $90-95 \%$ & 15 & 15.7 & $3.364 \pm 2.89$ & $4.526 \pm 2.94$ & $0.0447 \pm 0.038$ & $0.756 \pm 0.258$ & $0.353 \pm 0.313$ & $1.06 \pm 0.641$ & $0.859 \pm 0.787$ \\
\hline $95-100 \%$ & 15.7 & 20 & $2.071 \pm 1.78$ & $3.135 \pm 1.85$ & $0.0275 \pm 0.024$ & $0.838 \pm 0.246$ & $0.26 \pm 0.329$ & $0.947 \pm 0.449$ & $0.568 \pm 0.575$ \\
\hline $0-100 \%$ & 0 & 20 & $420.7 \pm 561$ & $115.4 \pm 117$ & $5.59 \pm 7.4$ & $0.429 \pm 0.275$ & $0.269 \pm 0.203$ & $2.9 \pm 2.35$ & $29 \pm 31.1$ \\
\hline
\end{tabular}


TABLE XIII. Various quantities for PbPb collisions at $\sqrt{s_{N N}}=39 \mathrm{TeV}$ obtained with the improved MCG model for centrality classes defined by slicing the impact parameter $(b)$ distribution. The mean and standard deviation of each quantity (denoted as rms) are given. Typical uncertainties, due to the MCG model parameters for each quantity at any centrality bin, can be read off from Fig. 12.

\begin{tabular}{|c|c|c|c|c|c|c|c|c|c|}
\hline Centrality & $b_{\min }(\mathrm{fm})$ & $b_{\max }(\mathrm{fm})$ & $\left\langle N_{\text {coll }}\right\rangle \pm \mathrm{rms}$ & $\left\langle N_{\text {part }}\right\rangle \pm \mathrm{rms}$ & $\left\langle T_{A A}\right\rangle \pm \mathrm{rms}\left(\mathrm{mb}^{-1}\right)$ & $\varepsilon_{2} \pm \mathrm{rms}$ & $\varepsilon_{3} \pm \mathrm{rms}$ & $L \pm \mathrm{rms}(\mathrm{fm})$ & $A_{\perp} \pm \mathrm{rms}\left(\mathrm{fm}^{2}\right)$ \\
\hline $0-5 \%$ & 0 & 3.55 & $2334 \pm 196$ & $390.9 \pm 14.8$ & $25.8 \pm 2.2$ & $0.0675 \pm 0.0362$ & $0.0808 \pm 0.0432$ & $5.34 \pm 2.97$ & $116 \pm 9.21$ \\
\hline $5-10 \%$ & 3.55 & 5.02 & $1817 \pm 150$ & $341 \pm 17.4$ & $20.1 \pm 1.7$ & $0.0991 \pm 0.0522$ & $0.109 \pm 0.057$ & $5.06 \pm 2.84$ & $94.2 \pm 6.8$ \\
\hline $15-20 \%$ & 6.15 & 7.11 & $1109 \pm 106$ & $250.3 \pm 16.6$ & $12.2 \pm 1.2$ & $0.18 \pm 0.0811$ & $0.151 \pm 0.0784$ & $4.52 \pm 2.57$ & $66.1 \pm 5.36$ \\
\hline $20-25 \%$ & 7.11 & 7.94 & $857.8 \pm 93$ & $212.6 \pm 16$ & $9.48 \pm 1$ & $0.219 \pm 0.093$ & $0.17 \pm 0.0883$ & $4.28 \pm 2.45$ & $55.7 \pm 4.99$ \\
\hline $25-30 \%$ & 7.94 & 8.7 & $655.9 \pm 82.5$ & $179.3 \pm 15.4$ & $7.25 \pm 0.91$ & $0.255 \pm 0.104$ & $0.189 \pm 0.0975$ & $4.03 \pm 2.33$ & $46.8 \pm 4.69$ \\
\hline $35-40 \%$ & 9.4 & 10 & $366.4 \pm 63.3$ & $124 \pm 14.3$ & $4.05 \pm 0.7$ & $0.323 \pm 0.126$ & $0.227 \pm 0.116$ & $3.56 \pm 2.1$ & $32.3 \pm 4.24$ \\
\hline $40-45 \%$ & 10 & 10.7 & $265.8 \pm 54$ & $101.3 \pm 13.8$ & $2.94 \pm 0.6$ & $0.355 \pm 0.137$ & $0.249 \pm 0.125$ & $3.33 \pm 2$ & $26.3 \pm 4.03$ \\
\hline $45-50 \%$ & 10.7 & 11.2 & $189 \pm 45$ & $81.63 \pm 13.1$ & $2.09 \pm 0.5$ & $0.386 \pm 0.148$ & $0.271 \pm 0.134$ & $3.11 \pm 1.9$ & $21.2 \pm 3.82$ \\
\hline $50-55 \%$ & 11.2 & 11.8 & $131 \pm 36.7$ & $64.58 \pm 12.4$ & $1.45 \pm 0.41$ & $0.418 \pm 0.16$ & $0.295 \pm 0.143$ & $2.89 \pm 1.8$ & $16.6 \pm 3.59$ \\
\hline $55-60 \%$ & 11.8 & 12.3 & $88.42 \pm 29.2$ & $49.99 \pm 11.5$ & $0.977 \pm 0.32$ & $0.451 \pm 0.172$ & $0.319 \pm 0.152$ & $2.68 \pm 1.71$ & $12.8 \pm 3.33$ \\
\hline $60-65 \%$ & 12.3 & 12.8 & $58.06 \pm 22.6$ & $37.76 \pm 10.6$ & $0.642 \pm 0.25$ & $0.485 \pm 0.184$ & $0.344 \pm 0.161$ & $2.45 \pm 1.6$ & $9.51 \pm 3.04$ \\
\hline $85-90 \%$ & 14.7 & 15.2 & $5.539 \pm 4.6$ & $6.606 \pm 4.28$ & $0.0612 \pm 0.051$ & $0.681 \pm 0.251$ & $0.393 \pm 0.273$ & $1.33 \pm 0.877$ & $1.46 \pm 1.22$ \\
\hline $90-95 \%$ & 15.2 & 15.9 & $3.517 \pm 3.1$ & $4.641 \pm 3.09$ & $0.0389 \pm 0.034$ & $0.74 \pm 0.263$ & $0.359 \pm 0.313$ & $1.18 \pm 0.696$ & $0.99 \pm 0.929$ \\
\hline $95-100 \%$ & 15.9 & 20 & $2.15 \pm 1.93$ & $3.204 \pm 1.99$ & $0.0238 \pm 0.021$ & $0.828 \pm 0.253$ & $0.267 \pm 0.332$ & $1.05 \pm 0.488$ & $0.655 \pm 0.682$ \\
\hline $0-100 \%$ & 0 & 20 & $494.1 \pm 668$ & $118.1 \pm 119$ & $5.46 \pm 7.4$ & $0.412 \pm 0.272$ & $0.265 \pm 0.201$ & $3.04 \pm 2.36$ & $31.8 \pm 33.7$ \\
\hline
\end{tabular}

TABLE XIV. Various quantities for $p \mathrm{~Pb}$ collisions at $\sqrt{s_{N N}}=5.02 \mathrm{TeV}$ obtained with the improved MCG model for centrality classes defined by slicing the impact parameter $(b)$ distribution. The mean and standard deviation of each quantity (denoted as rms) are given. Typical uncertainties, due to the MCG model parameters for each quantity at any centrality bin, can be read off from Fig. 13.

\begin{tabular}{|c|c|c|c|c|c|c|c|c|c|}
\hline Centrality & $b_{\min }(\mathrm{fm})$ & $b_{\max }(\mathrm{fm})$ & $\left\langle N_{\text {coll }}\right\rangle \pm \mathrm{rms}$ & $\left\langle N_{\text {part }}\right\rangle \pm \mathrm{rms}$ & $\left\langle T_{A A}\right\rangle \pm \mathrm{rms}\left(\mathrm{mb}^{-1}\right)$ & $\varepsilon_{2} \pm \mathrm{rms}$ & $\varepsilon_{3} \pm \mathrm{rms}$ & $L \pm \mathrm{rms}(\mathrm{fm})$ & $A_{\perp} \pm \mathrm{rms}\left(\mathrm{fm}^{2}\right)$ \\
\hline $0-5 \%$ & 0 & 1.82 & $13.68 \pm 3.51$ & $14.68 \pm 3.51$ & $0.202 \pm 0.052$ & $0.299 \pm 0.157$ & $0.328 \pm 0.168$ & $1.23 \pm 0.434$ & $1.64 \pm 0.123$ \\
\hline $5-10 \%$ & 1.82 & 2.58 & $13.11 \pm 3.4$ & $14.11 \pm 3.4$ & $0.194 \pm 0.05$ & $0.307 \pm 0.161$ & $0.337 \pm 0.171$ & $1.22 \pm 0.436$ & $1.63 \pm 0.135$ \\
\hline $15-20 \%$ & 3.16 & 3.65 & $11.83 \pm 3.18$ & $12.83 \pm 3.18$ & $0.175 \pm 0.047$ & $0.326 \pm 0.17$ & $0.356 \pm 0.179$ & $1.2 \pm 0.439$ & $1.61 \pm 0.161$ \\
\hline $20-25 \%$ & 3.65 & 4.08 & $11.13 \pm 3.07$ & $12.13 \pm 3.07$ & $0.165 \pm 0.045$ & $0.338 \pm 0.176$ & $0.369 \pm 0.184$ & $1.19 \pm 0.442$ & $1.6 \pm 0.179$ \\
\hline $25-30 \%$ & 4.08 & 4.47 & $10.36 \pm 2.96$ & $11.36 \pm 2.96$ & $0.153 \pm 0.044$ & $0.354 \pm 0.183$ & $0.384 \pm 0.19$ & $1.17 \pm 0.443$ & $1.58 \pm 0.201$ \\
\hline $35-40 \%$ & 4.83 & 5.16 & $8.646 \pm 2.7$ & $9.646 \pm 2.7$ & $0.128 \pm 0.04$ & $0.396 \pm 0.201$ & $0.422 \pm 0.203$ & $1.12 \pm 0.445$ & $1.51 \pm 0.26$ \\
\hline $40-45 \%$ & 5.16 & 5.47 & $7.721 \pm 2.57$ & $8.721 \pm 2.57$ & $0.114 \pm 0.038$ & $0.424 \pm 0.212$ & $0.445 \pm 0.21$ & $1.1 \pm 0.444$ & $1.46 \pm 0.299$ \\
\hline $45-50 \%$ & 5.47 & 5.77 & $6.766 \pm 2.41$ & $7.766 \pm 2.41$ & $0.1 \pm 0.036$ & $0.459 \pm 0.224$ & $0.467 \pm 0.218$ & $1.06 \pm 0.439$ & $1.39 \pm 0.344$ \\
\hline $50-55 \%$ & 5.77 & 6.05 & $5.836 \pm 2.25$ & $6.836 \pm 2.25$ & $0.0863 \pm 0.033$ & $0.498 \pm 0.236$ & $0.488 \pm 0.227$ & $1.03 \pm 0.433$ & $1.3 \pm 0.39$ \\
\hline $55-60 \%$ & 6.05 & 6.32 & $4.949 \pm 2.07$ & $5.949 \pm 2.07$ & $0.0732 \pm 0.031$ & $0.539 \pm 0.248$ & $0.504 \pm 0.24$ & $0.989 \pm 0.425$ & $1.19 \pm 0.432$ \\
\hline $60-65 \%$ & 6.32 & 6.58 & $4.132 \pm 1.87$ & $5.132 \pm 1.87$ & $0.0611 \pm 0.028$ & $0.586 \pm 0.261$ & $0.508 \pm 0.259$ & $0.952 \pm 0.412$ & $1.07 \pm 0.464$ \\
\hline $85-90 \%$ & 7.65 & 7.99 & $1.55 \pm 0.784$ & $2.55 \pm 0.784$ & $0.0229 \pm 0.012$ & $0.865 \pm 0.234$ & $0.26 \pm 0.348$ & $0.828 \pm 0.324$ & $0.467 \pm 0.412$ \\
\hline $90-95 \%$ & 7.99 & 8.49 & $1.287 \pm 0.556$ & $2.287 \pm 0.556$ & $0.019 \pm 0.0082$ & $0.923 \pm 0.188$ & $0.159 \pm 0.303$ & $0.816 \pm 0.307$ & $0.389 \pm 0.379$ \\
\hline $95-100 \%$ & 8.49 & 14.7 & $1.082 \pm 0.295$ & $2.082 \pm 0.295$ & $0.016 \pm 0.0044$ & $0.976 \pm 0.109$ & $0.0516 \pm 0.189$ & $0.805 \pm 0.291$ & $0.327 \pm 0.348$ \\
\hline $0-100 \%$ & 0 & 14.7 & $6.723 \pm 4.87$ & $7.723 \pm 4.87$ & $0.0994 \pm 0.072$ & $0.543 \pm 0.306$ & $0.378 \pm 0.271$ & $1.03 \pm 0.433$ & $1.17 \pm 0.574$ \\
\hline
\end{tabular}


TABLE XV. Various quantities for $p \mathrm{~Pb}$ collisions at $\sqrt{s_{N N}}=8.16 \mathrm{TeV}$ obtained with the improved MCG model for centrality classes defined by slicing the impact parameter $(b)$ distribution. The mean and standard deviation of each quantity (denoted as rms) are given. Typical uncertainties, due to the MCG model parameters for each quantity at any centrality bin, can be read off from Fig. 13.

\begin{tabular}{|c|c|c|c|c|c|c|c|c|c|}
\hline Centrality & $b_{\min }(\mathrm{fm})$ & $b_{\max }(\mathrm{fm})$ & $\left\langle N_{\text {coll }}\right\rangle \pm \mathrm{rms}$ & $\left\langle N_{\text {part }}\right\rangle \pm \mathrm{rms}$ & $\left\langle T_{A A}\right\rangle \pm \mathrm{rms}\left(\mathrm{mb}^{-1}\right)$ & $\varepsilon_{2} \pm \mathrm{rms}$ & $\varepsilon_{3} \pm \mathrm{rms}$ & $L \pm \mathrm{rms}(\mathrm{fm})$ & $A_{\perp} \pm \mathrm{rms}\left(\mathrm{fm}^{2}\right)$ \\
\hline $0-5 \%$ & 0 & 1.84 & $14.65 \pm 3.59$ & $15.65 \pm 3.59$ & $0.202 \pm 0.05$ & $0.286 \pm 0.151$ & $0.315 \pm 0.162$ & $1.29 \pm 0.445$ & $1.77 \pm 0.11$ \\
\hline $5-10 \%$ & 1.84 & 2.6 & $14.01 \pm 3.51$ & $15.01 \pm 3.51$ & $0.193 \pm 0.048$ & $0.295 \pm 0.155$ & $0.325 \pm 0.166$ & $1.28 \pm 0.447$ & $1.76 \pm 0.122$ \\
\hline $15-20 \%$ & 3.19 & 3.68 & $12.62 \pm 3.27$ & $13.62 \pm 3.27$ & $0.174 \pm 0.045$ & $0.314 \pm 0.164$ & $0.344 \pm 0.174$ & $1.26 \pm 0.451$ & $1.74 \pm 0.152$ \\
\hline $20-25 \%$ & 3.68 & 4.11 & $11.87 \pm 3.16$ & $12.87 \pm 3.16$ & $0.164 \pm 0.044$ & $0.326 \pm 0.17$ & $0.357 \pm 0.179$ & $1.24 \pm 0.453$ & $1.73 \pm 0.169$ \\
\hline $35-40 \%$ & 4.87 & 5.2 & $9.116 \pm 2.78$ & $10.12 \pm 2.78$ & $0.126 \pm 0.038$ & $0.384 \pm 0.196$ & $0.413 \pm 0.199$ & $1.18 \pm 0.459$ & $1.64 \pm 0.261$ \\
\hline $40-45 \%$ & 5.2 & 5.52 & $8.091 \pm 2.62$ & $9.091 \pm 2.62$ & $0.112 \pm 0.036$ & $0.413 \pm 0.207$ & $0.437 \pm 0.207$ & $1.14 \pm 0.456$ & $1.59 \pm 0.303$ \\
\hline $45-50 \%$ & 5.52 & 5.82 & $7.06 \pm 2.46$ & $8.06 \pm 2.46$ & $0.0974 \pm 0.034$ & $0.449 \pm 0.219$ & $0.461 \pm 0.215$ & $1.11 \pm 0.454$ & $1.51 \pm 0.355$ \\
\hline $50-55 \%$ & 5.82 & 6.1 & $6.064 \pm 2.29$ & $7.064 \pm 2.29$ & $0.0836 \pm 0.032$ & $0.486 \pm 0.232$ & $0.485 \pm 0.224$ & $1.07 \pm 0.448$ & $1.42 \pm 0.406$ \\
\hline $55-60 \%$ & 6.1 & 6.38 & $5.108 \pm 2.1$ & $6.108 \pm 2.1$ & $0.0705 \pm 0.029$ & $0.529 \pm 0.246$ & $0.503 \pm 0.237$ & $1.03 \pm 0.438$ & $1.29 \pm 0.456$ \\
\hline $60-65 \%$ & 6.38 & 6.64 & $4.241 \pm 1.9$ & $5.241 \pm 1.9$ & $0.0585 \pm 0.026$ & $0.575 \pm 0.258$ & $0.51 \pm 0.256$ & $0.992 \pm 0.425$ & $1.15 \pm 0.493$ \\
\hline $85-90 \%$ & 7.71 & 8.05 & $1.556 \pm 0.787$ & $2.556 \pm 0.787$ & $0.0215 \pm 0.011$ & $0.862 \pm 0.237$ & $0.265 \pm 0.351$ & $0.861 \pm 0.335$ & $0.496 \pm 0.438$ \\
\hline $90-95 \%$ & 8.05 & 8.55 & $1.291 \pm 0.56$ & $2.291 \pm 0.56$ & $0.0178 \pm 0.0077$ & $0.921 \pm 0.191$ & $0.162 \pm 0.306$ & $0.849 \pm 0.316$ & $0.41 \pm 0.402$ \\
\hline $95-100 \%$ & 8.55 & 14.8 & $1.083 \pm 0.296$ & $2.083 \pm 0.296$ & $0.0149 \pm 0.0041$ & $0.976 \pm 0.11$ & $0.0517 \pm 0.189$ & $0.838 \pm 0.302$ & $0.343 \pm 0.369$ \\
\hline $0-100 \%$ & 0 & 14.8 & $7.085 \pm 5.19$ & $8.085 \pm 5.19$ & $0.0977 \pm 0.072$ & $0.534 \pm 0.306$ & $0.374 \pm 0.269$ & $1.08 \pm 0.448$ & $1.26 \pm 0.618$ \\
\hline
\end{tabular}

TABLE XVI. Various quantities for $p \mathrm{~Pb}$ collisions at $\sqrt{s_{N N}}=8.8 \mathrm{TeV}$ obtained with the improved MCG model for centrality classes defined by slicing the impact parameter $(b)$ distribution. The mean and standard deviation of each quantity (denoted as rms) are given. Typical uncertainties, due to the MCG model parameters for each quantity at any centrality bin, can be read off from Fig. 13.

\begin{tabular}{|c|c|c|c|c|c|c|c|c|c|}
\hline Centrality & $b_{\min }(\mathrm{fm})$ & $b_{\max }(\mathrm{fm})$ & $\left\langle N_{\text {coll }}\right\rangle \pm \mathrm{rms}$ & $\left\langle N_{\text {part }}\right\rangle \pm \mathrm{rms}$ & $\left\langle T_{A A}\right\rangle \pm \mathrm{rms}\left(\mathrm{mb}^{-1}\right)$ & $\varepsilon_{2} \pm \mathrm{rms}$ & $\varepsilon_{3} \pm \mathrm{rms}$ & $L \pm \mathrm{rms}(\mathrm{fm})$ & $A_{\perp} \pm \mathrm{rms}\left(\mathrm{fm}^{2}\right)$ \\
\hline $0-5 \%$ & 0 & 1.84 & $14.83 \pm 3.63$ & $15.83 \pm 3.63$ & $0.202 \pm 0.05$ & $0.285 \pm 0.15$ & $0.314 \pm 0.161$ & $1.3 \pm 0.447$ & $1.79 \pm 0.109$ \\
\hline $5-10 \%$ & 1.84 & 2.6 & $14.18 \pm 3.52$ & $15.18 \pm 3.52$ & $0.193 \pm 0.048$ & $0.293 \pm 0.154$ & $0.322 \pm 0.165$ & $1.29 \pm 0.447$ & $1.79 \pm 0.12$ \\
\hline $15-20 \%$ & 3.19 & 3.68 & $12.75 \pm 3.29$ & $13.75 \pm 3.29$ & $0.174 \pm 0.045$ & $0.312 \pm 0.164$ & $0.342 \pm 0.173$ & $1.26 \pm 0.454$ & $1.77 \pm 0.149$ \\
\hline $20-25 \%$ & 3.68 & 4.12 & $11.97 \pm 3.16$ & $12.97 \pm 3.16$ & $0.163 \pm 0.043$ & $0.324 \pm 0.169$ & $0.355 \pm 0.179$ & $1.25 \pm 0.457$ & $1.75 \pm 0.168$ \\
\hline $25-30 \%$ & 4.12 & 4.51 & $11.11 \pm 3.04$ & $12.11 \pm 3.04$ & $0.152 \pm 0.041$ & $0.34 \pm 0.177$ & $0.369 \pm 0.184$ & $1.23 \pm 0.457$ & $1.73 \pm 0.193$ \\
\hline $35-40 \%$ & 4.87 & 5.21 & $9.179 \pm 2.78$ & $10.18 \pm 2.78$ & $0.125 \pm 0.038$ & $0.383 \pm 0.195$ & $0.411 \pm 0.199$ & $1.18 \pm 0.461$ & $1.67 \pm 0.259$ \\
\hline $40-45 \%$ & 5.21 & 5.53 & $8.151 \pm 2.64$ & $9.151 \pm 2.64$ & $0.111 \pm 0.036$ & $0.411 \pm 0.206$ & $0.435 \pm 0.207$ & $1.15 \pm 0.459$ & $1.61 \pm 0.305$ \\
\hline $45-50 \%$ & 5.53 & 5.83 & $7.113 \pm 2.47$ & $8.113 \pm 2.47$ & $0.097 \pm 0.034$ & $0.446 \pm 0.218$ & $0.461 \pm 0.215$ & $1.11 \pm 0.455$ & $1.54 \pm 0.355$ \\
\hline $50-55 \%$ & 5.83 & 6.11 & $6.082 \pm 2.29$ & $7.082 \pm 2.29$ & $0.083 \pm 0.031$ & $0.487 \pm 0.232$ & $0.481 \pm 0.224$ & $1.07 \pm 0.45$ & $1.43 \pm 0.41$ \\
\hline $55-60 \%$ & 6.11 & 6.38 & $5.148 \pm 2.11$ & $6.148 \pm 2.11$ & $0.0702 \pm 0.029$ & $0.527 \pm 0.245$ & $0.501 \pm 0.237$ & $1.03 \pm 0.44$ & $1.31 \pm 0.459$ \\
\hline $60-65 \%$ & 6.38 & 6.65 & $4.266 \pm 1.91$ & $5.266 \pm 1.91$ & $0.0582 \pm 0.026$ & $0.574 \pm 0.258$ & $0.509 \pm 0.256$ & $0.999 \pm 0.427$ & $1.16 \pm 0.499$ \\
\hline $85-90 \%$ & 7.72 & 8.06 & $1.557 \pm 0.79$ & $2.557 \pm 0.79$ & $0.0212 \pm 0.011$ & $0.863 \pm 0.236$ & $0.263 \pm 0.349$ & $0.869 \pm 0.338$ & $0.498 \pm 0.443$ \\
\hline $90-95 \%$ & 8.06 & 8.56 & $1.294 \pm 0.564$ & $2.294 \pm 0.564$ & $0.0177 \pm 0.0077$ & $0.921 \pm 0.191$ & $0.163 \pm 0.306$ & $0.854 \pm 0.319$ & $0.415 \pm 0.407$ \\
\hline $95-100 \%$ & 8.56 & 14.4 & $1.08 \pm 0.292$ & $2.08 \pm 0.292$ & $0.0147 \pm 0.004$ & $0.977 \pm 0.109$ & $0.0508 \pm 0.188$ & $0.843 \pm 0.302$ & $0.345 \pm 0.371$ \\
\hline $0-100 \%$ & 0 & 14.4 & $7.146 \pm 5.25$ & $8.146 \pm 5.25$ & $0.0975 \pm 0.072$ & $0.532 \pm 0.307$ & $0.373 \pm 0.269$ & $1.08 \pm 0.45$ & $1.27 \pm 0.626$ \\
\hline
\end{tabular}


TABLE XVII. Various quantities for $p \mathrm{~Pb}$ collisions at $\sqrt{s_{N N}}=17 \mathrm{TeV}$ obtained with the improved MCG model for centrality classes defined by slicing the impact parameter $(b)$ distribution. The mean and standard deviation of each quantity (denoted as rms) are given. Typical uncertainties, due to the MCG model parameters for each quantity at any centrality bin, can be read off from Fig. 13.

\begin{tabular}{|c|c|c|c|c|c|c|c|c|c|}
\hline Centrality & $b_{\min }(\mathrm{fm})$ & $b_{\max }(\mathrm{fm})$ & $\left\langle N_{\text {coll }}\right\rangle \pm \mathrm{rms}$ & $\left\langle N_{\text {part }}\right\rangle \pm \mathrm{rms}$ & $\left\langle T_{A A}\right\rangle \pm \mathrm{rms}\left(\mathrm{mb}^{-1}\right)$ & $\varepsilon_{2} \pm \mathrm{rms}$ & $\varepsilon_{3} \pm \mathrm{rms}$ & $L \pm \mathrm{rms}(\mathrm{fm})$ & $A_{\perp} \pm \mathrm{rms}\left(\mathrm{fm}^{2}\right)$ \\
\hline $0-5 \%$ & 0 & 1.86 & $16.26 \pm 3.78$ & $17.26 \pm 3.78$ & $0.202 \pm 0.047$ & $0.27 \pm 0.143$ & $0.297 \pm 0.153$ & $1.38 \pm 0.461$ & $1.99 \pm 0.0941$ \\
\hline $5-10 \%$ & 1.86 & 2.63 & $15.54 \pm 3.65$ & $16.54 \pm 3.65$ & $0.193 \pm 0.045$ & $0.278 \pm 0.146$ & $0.305 \pm 0.157$ & $1.37 \pm 0.464$ & $1.98 \pm 0.103$ \\
\hline $15-20 \%$ & 3.23 & 3.73 & $13.92 \pm 3.42$ & $14.92 \pm 3.42$ & $0.173 \pm 0.042$ & $0.296 \pm 0.155$ & $0.327 \pm 0.166$ & $1.34 \pm 0.47$ & $1.96 \pm 0.135$ \\
\hline $20-25 \%$ & 3.73 & 4.17 & $13.01 \pm 3.27$ & $14.01 \pm 3.27$ & $0.161 \pm 0.041$ & $0.307 \pm 0.16$ & $0.34 \pm 0.172$ & $1.33 \pm 0.472$ & $1.95 \pm 0.156$ \\
\hline $25-30 \%$ & 4.17 & 4.56 & $12.02 \pm 3.14$ & $13.02 \pm 3.14$ & $0.149 \pm 0.039$ & $0.324 \pm 0.169$ & $0.355 \pm 0.178$ & $1.31 \pm 0.473$ & $1.93 \pm 0.181$ \\
\hline $35-40 \%$ & 4.93 & 5.27 & $9.849 \pm 2.87$ & $10.85 \pm 2.87$ & $0.122 \pm 0.036$ & $0.368 \pm 0.188$ & $0.398 \pm 0.194$ & $1.25 \pm 0.478$ & $1.86 \pm 0.256$ \\
\hline $40-45 \%$ & 5.27 & 5.59 & $8.701 \pm 2.7$ & $9.701 \pm 2.7$ & $0.108 \pm 0.034$ & $0.398 \pm 0.2$ & $0.424 \pm 0.202$ & $1.22 \pm 0.478$ & $1.8 \pm 0.31$ \\
\hline $45-50 \%$ & 5.59 & 5.89 & $7.539 \pm 2.53$ & $8.539 \pm 2.53$ & $0.0935 \pm 0.031$ & $0.433 \pm 0.212$ & $0.451 \pm 0.212$ & $1.18 \pm 0.473$ & $1.72 \pm 0.369$ \\
\hline $50-55 \%$ & 5.89 & 6.18 & $6.412 \pm 2.36$ & $7.412 \pm 2.36$ & $0.0796 \pm 0.029$ & $0.473 \pm 0.226$ & $0.477 \pm 0.221$ & $1.14 \pm 0.468$ & $1.6 \pm 0.437$ \\
\hline $55-60 \%$ & 6.18 & 6.46 & $5.376 \pm 2.17$ & $6.376 \pm 2.17$ & $0.0667 \pm 0.027$ & $0.517 \pm 0.24$ & $0.497 \pm 0.233$ & $1.09 \pm 0.459$ & $1.46 \pm 0.496$ \\
\hline $60-65 \%$ & 6.46 & 6.73 & $4.425 \pm 1.95$ & $5.425 \pm 1.95$ & $0.0549 \pm 0.024$ & $0.562 \pm 0.255$ & $0.508 \pm 0.251$ & $1.05 \pm 0.447$ & $1.29 \pm 0.539$ \\
\hline $85-90 \%$ & 7.8 & 8.14 & $1.575 \pm 0.801$ & $2.575 \pm 0.801$ & $0.0195 \pm 0.0099$ & $0.857 \pm 0.24$ & $0.272 \pm 0.353$ & $0.917 \pm 0.354$ & $0.54 \pm 0.483$ \\
\hline $90-95 \%$ & 8.14 & 8.64 & $1.295 \pm 0.561$ & $2.295 \pm 0.561$ & $0.0161 \pm 0.007$ & $0.919 \pm 0.193$ & $0.166 \pm 0.309$ & $0.902 \pm 0.335$ & $0.442 \pm 0.439$ \\
\hline $95-100 \%$ & 8.64 & 14.9 & $1.084 \pm 0.298$ & $2.084 \pm 0.298$ & $0.0134 \pm 0.0037$ & $0.976 \pm 0.112$ & $0.0529 \pm 0.191$ & $0.891 \pm 0.319$ & $0.371 \pm 0.403$ \\
\hline $0-100 \%$ & 0 & 14.9 & $7.677 \pm 5.72$ & $8.677 \pm 5.72$ & $0.0952 \pm 0.071$ & $0.52 \pm 0.308$ & $0.367 \pm 0.266$ & $1.14 \pm 0.47$ & $1.41 \pm 0.693$ \\
\hline
\end{tabular}

TABLE XVIII. Various quantities for $p \mathrm{~Pb}$ collisions at $\sqrt{s_{N N}}=63 \mathrm{TeV}$ obtained with the improved MCG model for centrality classes defined by slicing the impact parameter $(b)$ distribution. The mean and standard deviation of each quantity (denoted as rms) are given. Typical uncertainties, due to the MCG model parameters for each quantity at any centrality bin, can be read off from Fig. 13.

\begin{tabular}{|c|c|c|c|c|c|c|c|c|c|}
\hline Centrality & $b_{\min }(\mathrm{fm})$ & $b_{\max }(\mathrm{fm})$ & $\left\langle N_{\text {coll }}\right\rangle \pm \mathrm{rms}$ & $\left\langle N_{\text {part }}\right\rangle \pm \mathrm{rms}$ & $\left\langle T_{A A}\right\rangle \pm \mathrm{rms}\left(\mathrm{mb}^{-1}\right)$ & $\varepsilon_{2} \pm \mathrm{rms}$ & $\varepsilon_{3} \pm \mathrm{rms}$ & $L \pm \mathrm{rms}(\mathrm{fm})$ & $A_{\perp} \pm \operatorname{rms}\left(\mathrm{fm}^{2}\right)$ \\
\hline $0-5 \%$ & 0 & 1.91 & $19.37 \pm 4.11$ & $20.37 \pm 4.11$ & $0.201 \pm 0.043$ & $0.244 \pm 0.129$ & $0.27 \pm 0.139$ & $1.54 \pm 0.493$ & $2.4 \pm 0.0711$ \\
\hline $5-10 \%$ & 1.91 & 2.7 & $18.45 \pm 3.96$ & $19.45 \pm 3.96$ & $0.191 \pm 0.041$ & $0.251 \pm 0.133$ & $0.277 \pm 0.144$ & $1.53 \pm 0.494$ & $2.39 \pm 0.0801$ \\
\hline $15-20 \%$ & 3.31 & 3.81 & $16.39 \pm 3.64$ & $17.39 \pm 3.64$ & $0.17 \pm 0.038$ & $0.27 \pm 0.141$ & $0.297 \pm 0.153$ & $1.5 \pm 0.497$ & $2.38 \pm 0.105$ \\
\hline $20-25 \%$ & 3.81 & 4.26 & $15.24 \pm 3.5$ & $16.24 \pm 3.5$ & $0.158 \pm 0.036$ & $0.281 \pm 0.147$ & $0.312 \pm 0.159$ & $1.49 \pm 0.503$ & $2.37 \pm 0.128$ \\
\hline $25-30 \%$ & 4.26 & 4.67 & $13.97 \pm 3.34$ & $14.97 \pm 3.34$ & $0.145 \pm 0.035$ & $0.298 \pm 0.155$ & $0.328 \pm 0.166$ & $1.46 \pm 0.505$ & $2.35 \pm 0.156$ \\
\hline $35-40 \%$ & 5.04 & 5.39 & $11.19 \pm 3.02$ & $12.19 \pm 3.02$ & $0.116 \pm 0.031$ & $0.346 \pm 0.175$ & $0.374 \pm 0.184$ & $1.39 \pm 0.512$ & $2.28 \pm 0.251$ \\
\hline $40-45 \%$ & 5.39 & 5.72 & $9.76 \pm 2.83$ & $10.76 \pm 2.83$ & $0.101 \pm 0.029$ & $0.377 \pm 0.188$ & $0.403 \pm 0.193$ & $1.35 \pm 0.513$ & $2.21 \pm 0.318$ \\
\hline $45-50 \%$ & 5.72 & 6.03 & $8.359 \pm 2.66$ & $9.359 \pm 2.66$ & $0.0866 \pm 0.028$ & $0.413 \pm 0.201$ & $0.433 \pm 0.204$ & $1.3 \pm 0.508$ & $2.1 \pm 0.403$ \\
\hline $50-55 \%$ & 6.03 & 6.32 & $7.046 \pm 2.45$ & $8.046 \pm 2.45$ & $0.073 \pm 0.025$ & $0.451 \pm 0.214$ & $0.462 \pm 0.215$ & $1.26 \pm 0.504$ & $1.96 \pm 0.49$ \\
\hline $55-60 \%$ & 6.32 & 6.6 & $5.837 \pm 2.26$ & $6.837 \pm 2.26$ & $0.0605 \pm 0.023$ & $0.492 \pm 0.23$ & $0.488 \pm 0.227$ & $1.21 \pm 0.494$ & $1.78 \pm 0.571$ \\
\hline $60-65 \%$ & 6.6 & 6.88 & $4.771 \pm 2.03$ & $5.771 \pm 2.03$ & $0.0494 \pm 0.021$ & $0.534 \pm 0.246$ & $0.508 \pm 0.243$ & $1.17 \pm 0.484$ & $1.56 \pm 0.63$ \\
\hline $85-90 \%$ & 7.96 & 8.31 & $1.599 \pm 0.819$ & $2.599 \pm 0.819$ & $0.0166 \pm 0.0085$ & $0.849 \pm 0.246$ & $0.283 \pm 0.357$ & $1.02 \pm 0.385$ & $0.617 \pm 0.558$ \\
\hline $90-95 \%$ & 8.31 & 8.8 & $1.309 \pm 0.578$ & $2.309 \pm 0.578$ & $0.0136 \pm 0.006$ & $0.915 \pm 0.198$ & $0.173 \pm 0.314$ & $1 \pm 0.366$ & $0.504 \pm 0.51$ \\
\hline $95-100 \%$ & 8.8 & 14.9 & $1.085 \pm 0.301$ & $2.085 \pm 0.301$ & $0.0112 \pm 0.0031$ & $0.975 \pm 0.114$ & $0.0546 \pm 0.195$ & $0.988 \pm 0.35$ & $0.419 \pm 0.466$ \\
\hline $0-100 \%$ & 0 & 14.9 & $8.789 \pm 6.74$ & $9.789 \pm 6.74$ & $0.0911 \pm 0.07$ & $0.5 \pm 0.309$ & $0.357 \pm 0.263$ & $1.27 \pm 0.509$ & $1.71 \pm 0.839$ \\
\hline
\end{tabular}


TABLE XIX. Various quantities for XeXe collisions at $\sqrt{s_{N N}}=5.44 \mathrm{TeV}$ obtained with the improved MCG model for centrality classes defined by slicing the impact parameter $(b)$ distribution. The mean and standard deviation of each quantity (denoted as rms) are given. Deformed profile for Xe was used.

\begin{tabular}{|c|c|c|c|c|c|c|c|c|c|}
\hline Centrality & $b_{\min }(\mathrm{fm})$ & $b_{\max }(\mathrm{fm})$ & $\left\langle N_{\text {coll }}\right\rangle \pm \mathrm{rms}$ & $\left\langle N_{\text {part }}\right\rangle \pm \mathrm{rms}$ & $\left\langle T_{A A}\right\rangle \pm \mathrm{rms}\left(\mathrm{mb}^{-1}\right)$ & $\varepsilon_{2} \pm \mathrm{rms}$ & $\varepsilon_{3} \pm \mathrm{rms}$ & $L \pm \mathrm{rms}(\mathrm{fm})$ & $A_{\perp} \pm \mathrm{rms}\left(\mathrm{fm}^{2}\right)$ \\
\hline $0-5 \%$ & 0 & 3.01 & $942.5 \pm 92.1$ & $236.5 \pm 10$ & $13.8 \pm 1.3$ & $0.116 \pm 0.06$ & $0.112 \pm 0.059$ & $4.33 \pm 2.52$ & $66.2 \pm 5.39$ \\
\hline $5-10 \%$ & 3.01 & 4.26 & $734.1 \pm 72.8$ & $206.1 \pm 11.7$ & $10.7 \pm 1.1$ & $0.141 \pm 0.0734$ & $0.145 \pm 0.0748$ & $4.11 \pm 2.42$ & $53.9 \pm 4.56$ \\
\hline $15-20 \%$ & 5.22 & 6.02 & $443.9 \pm 55.5$ & $151.1 \pm 12.4$ & $6.49 \pm 0.81$ & $0.207 \pm 0.103$ & $0.199 \pm 0.101$ & $3.67 \pm 2.23$ & $37.2 \pm 3.96$ \\
\hline $20-25 \%$ & 6.02 & 6.73 & $341.7 \pm 50.8$ & $127.9 \pm 12.6$ & $5 \pm 0.74$ & $0.243 \pm 0.117$ & $0.223 \pm 0.113$ & $3.45 \pm 2.13$ & $30.9 \pm 3.82$ \\
\hline $25-30 \%$ & 6.73 & 7.38 & $260.5 \pm 46.2$ & $107.4 \pm 12.6$ & $3.81 \pm 0.68$ & $0.278 \pm 0.13$ & $0.247 \pm 0.124$ & $3.24 \pm 2.03$ & $25.6 \pm 3.68$ \\
\hline $35-40 \%$ & 7.97 & 8.52 & $145.5 \pm 36.8$ & $73.53 \pm 12.4$ & $2.13 \pm 0.54$ & $0.349 \pm 0.156$ & $0.295 \pm 0.145$ & $2.85 \pm 1.86$ & $17.1 \pm 3.41$ \\
\hline $40-45 \%$ & 8.52 & 9.04 & $106.5 \pm 31.7$ & $59.75 \pm 12.1$ & $1.56 \pm 0.46$ & $0.386 \pm 0.168$ & $0.32 \pm 0.154$ & $2.64 \pm 1.77$ & $13.7 \pm 3.25$ \\
\hline $45-50 \%$ & 9.04 & 9.53 & $76.83 \pm 26.8$ & $47.94 \pm 11.6$ & $1.12 \pm 0.39$ & $0.424 \pm 0.18$ & $0.343 \pm 0.162$ & $2.45 \pm 1.69$ & $10.8 \pm 3.05$ \\
\hline $50-55 \%$ & 9.53 & 9.99 & $54.64 \pm 22.1$ & $37.9 \pm 10.9$ & $0.799 \pm 0.32$ & $0.463 \pm 0.191$ & $0.366 \pm 0.17$ & $2.26 \pm 1.6$ & $8.39 \pm 2.81$ \\
\hline $55-60 \%$ & 9.99 & 10.4 & $38.28 \pm 18$ & $29.43 \pm 10.1$ & $0.56 \pm 0.26$ & $0.504 \pm 0.202$ & $0.386 \pm 0.178$ & $2.05 \pm 1.5$ & $6.4 \pm 2.55$ \\
\hline $60-65 \%$ & 10.4 & 10.9 & $26.61 \pm 14.4$ & $22.56 \pm 9.17$ & $0.389 \pm 0.21$ & $0.545 \pm 0.211$ & $0.401 \pm 0.186$ & $1.85 \pm 1.39$ & $4.8 \pm 2.27$ \\
\hline $85-90 \%$ & 12.5 & 13.1 & $4.232 \pm 3.64$ & $5.422 \pm 3.6$ & $0.0619 \pm 0.053$ & $0.74 \pm 0.25$ & $0.375 \pm 0.295$ & $1.06 \pm 0.706$ & $1.03 \pm 0.891$ \\
\hline $90-95 \%$ & 13.1 & 13.8 & $2.967 \pm 2.58$ & $4.116 \pm 2.67$ & $0.0434 \pm 0.038$ & $0.786 \pm 0.252$ & $0.333 \pm 0.319$ & $0.965 \pm 0.568$ & $0.759 \pm 0.697$ \\
\hline $95-100 \%$ & 13.8 & 20 & $1.95 \pm 1.64$ & $3.007 \pm 1.72$ & $0.0285 \pm 0.024$ & $0.857 \pm 0.235$ & $0.241 \pm 0.324$ & $0.88 \pm 0.414$ & $0.533 \pm 0.524$ \\
\hline $0-100 \%$ & 0 & 20 & $199.6 \pm 270$ & $71.26 \pm 72$ & $2.92 \pm 3.9$ & $0.455 \pm 0.288$ & $0.303 \pm 0.215$ & $2.42 \pm 2.01$ & $17.6 \pm 19.3$ \\
\hline
\end{tabular}

TABLE XX. Various quantities for AuAu collisions at $\sqrt{s_{N N}}=0.2 \mathrm{TeV}$ obtained with the improved MCG model for centrality classes defined by slicing the impact parameter $(b)$ distribution. The mean and standard deviation of each quantity (denoted as rms) are given. Deformed profile for Au was used.

\begin{tabular}{|c|c|c|c|c|c|c|c|c|c|}
\hline Centrality & $b_{\min }(\mathrm{fm})$ & $b_{\max }(\mathrm{fm})$ & $\left\langle N_{\text {coll }}\right\rangle \pm \mathrm{rms}$ & $\left\langle N_{\text {part }}\right\rangle \pm \mathrm{rms}$ & $\left\langle T_{A A}\right\rangle \pm \mathrm{rms}\left(\mathrm{mb}^{-1}\right)$ & $\varepsilon_{2} \pm \mathrm{rms}$ & $\varepsilon_{3} \pm \mathrm{rms}$ & $L \pm \mathrm{rms}(\mathrm{fm})$ & $A_{\perp} \pm \mathrm{rms}\left(\mathrm{fm}^{2}\right)$ \\
\hline $5-10 \%$ & 3.31 & 4.68 & $831.4 \pm 72.1$ & $298.1 \pm 17$ & $20 \pm 1.7$ & $0.14 \pm 0.0704$ & $0.122 \pm 0.0633$ & $4.43 \pm 2.76$ & $54.6 \pm 4.05$ \\
\hline $15-20 \%$ & 5.73 & 6.61 & $523 \pm 54.4$ & $213.8 \pm 15.4$ & $12.6 \pm 1.3$ & $0.231 \pm 0.0985$ & $0.164 \pm 0.0846$ & $3.86 \pm 2.48$ & $37.5 \pm 3.29$ \\
\hline $20-25 \%$ & 6.61 & 7.39 & $412 \pm 49.5$ & $180.1 \pm 14.9$ & $9.9 \pm 1.2$ & $0.273 \pm 0.11$ & $0.184 \pm 0.0949$ & $3.6 \pm 2.36$ & $31.1 \pm 3.11$ \\
\hline $25-30 \%$ & 7.39 & 8.1 & $321.1 \pm 45.3$ & $150.8 \pm 14.6$ & $7.72 \pm 1.1$ & $0.313 \pm 0.121$ & $0.205 \pm 0.105$ & $3.35 \pm 2.24$ & $25.6 \pm 2.96$ \\
\hline $40-45 \%$ & 9.35 & 9.92 & $139.9 \pm 32.5$ & $83.36 \pm 13.4$ & $3.36 \pm 0.78$ & $0.418 \pm 0.154$ & $0.277 \pm 0.139$ & $2.64 \pm 1.91$ & $13.5 \pm 2.54$ \\
\hline $45-50 \%$ & 9.92 & 10.5 & $102.4 \pm 27.8$ & $66.65 \pm 12.7$ & $2.46 \pm 0.67$ & $0.452 \pm 0.166$ & $0.305 \pm 0.151$ & $2.41 \pm 1.8$ & $10.6 \pm 2.37$ \\
\hline $50-55 \%$ & 10.5 & 11 & $73.35 \pm 23.4$ & $52.37 \pm 11.9$ & $1.76 \pm 0.56$ & $0.485 \pm 0.178$ & $0.337 \pm 0.163$ & $2.18 \pm 1.69$ & $8.17 \pm 2.17$ \\
\hline $55-60 \%$ & 11 & 11.5 & $51.45 \pm 19.2$ & $40.39 \pm 11$ & $1.24 \pm 0.46$ & $0.522 \pm 0.19$ & $0.367 \pm 0.174$ & $1.95 \pm 1.57$ & $6.15 \pm 1.96$ \\
\hline $60-65 \%$ & 11.5 & 11.9 & $35.33 \pm 15.4$ & $30.5 \pm 9.95$ & $0.849 \pm 0.37$ & $0.56 \pm 0.202$ & $0.397 \pm 0.184$ & $1.72 \pm 1.43$ & $4.53 \pm 1.73$ \\
\hline $85-90 \%$ & 13.7 & 14.2 & $4.426 \pm 3.49$ & $5.852 \pm 3.67$ & $0.106 \pm 0.084$ & $0.767 \pm 0.233$ & $0.378 \pm 0.283$ & $0.828 \pm 0.624$ & $0.743 \pm 0.604$ \\
\hline $90-95 \%$ & 14.2 & 14.9 & $2.949 \pm 2.38$ & $4.216 \pm 2.6$ & $0.0709 \pm 0.057$ & $0.81 \pm 0.235$ & $0.329 \pm 0.31$ & $0.739 \pm 0.48$ & $0.517 \pm 0.454$ \\
\hline $95-100 \%$ & 14.9 & 20 & $1.867 \pm 1.43$ & $2.957 \pm 1.57$ & $0.0449 \pm 0.034$ & $0.874 \pm 0.218$ & $0.231 \pm 0.316$ & $0.665 \pm 0.335$ & $0.345 \pm 0.327$ \\
\hline $0-100 \%$ & 0 & 20 & $235.1 \pm 304$ & $100.9 \pm 105$ & $5.65 \pm 7.3$ & $0.477 \pm 0.284$ & $0.285 \pm 0.213$ & $2.4 \pm 2.24$ & $17.6 \pm 19.6$ \\
\hline
\end{tabular}


TABLE XXI. Various quantities for $\mathrm{CuCu}$ collisions at $\sqrt{s_{N N}}=0.2 \mathrm{TeV}$ obtained with the improved MCG model for centrality classes defined by slicing the impact parameter $(b)$ distribution. The mean and standard deviation of each quantity (denoted as rms) are given. Deformed profile for $\mathrm{Cu}$ was used.

\begin{tabular}{|c|c|c|c|c|c|c|c|c|c|}
\hline Centrality & $b_{\min }(\mathrm{fm})$ & $b_{\max }(\mathrm{fm})$ & $\left\langle N_{\text {coll }}\right\rangle \pm \mathrm{rms}$ & $\left\langle N_{\text {part }}\right\rangle \pm \mathrm{rms}$ & $\left\langle T_{A A}\right\rangle \pm \mathrm{rms}\left(\mathrm{mb}^{-1}\right)$ & $\varepsilon_{2} \pm \mathrm{rms}$ & $\varepsilon_{3} \pm \mathrm{rms}$ & $L \pm \mathrm{rms}(\mathrm{fm})$ & $A_{\perp} \pm \mathrm{rms}\left(\mathrm{fm}^{2}\right)$ \\
\hline $0-5 \%$ & 0 & 2.34 & $203.6 \pm 24.9$ & $106.5 \pm 6.21$ & $4.9 \pm 0.6$ & $0.164 \pm 0.0849$ & $0.181 \pm 0.0933$ & $3.12 \pm 2.08$ & $21.2 \pm 2.1$ \\
\hline $5-10 \%$ & 2.34 & 3.31 & $162.9 \pm 20.6$ & $91.68 \pm 6.41$ & $3.91 \pm 0.5$ & $0.199 \pm 0.102$ & $0.218 \pm 0.11$ & $2.92 \pm 2$ & $17.4 \pm 1.84$ \\
\hline $15-20 \%$ & 4.06 & 4.68 & $103.7 \pm 16.3$ & $66.83 \pm 6.65$ & $2.49 \pm 0.39$ & $0.274 \pm 0.135$ & $0.282 \pm 0.138$ & $2.54 \pm 1.83$ & $11.9 \pm 1.64$ \\
\hline $20-25 \%$ & 4.68 & 5.24 & $82.13 \pm 15$ & $56.58 \pm 6.78$ & $1.97 \pm 0.36$ & $0.313 \pm 0.149$ & $0.312 \pm 0.151$ & $2.36 \pm 1.74$ & $9.86 \pm 1.59$ \\
\hline $25-30 \%$ & 5.24 & 5.73 & $64.7 \pm 13.8$ & $47.63 \pm 6.86$ & $1.56 \pm 0.33$ & $0.353 \pm 0.164$ & $0.34 \pm 0.162$ & $2.19 \pm 1.66$ & $8.1 \pm 1.54$ \\
\hline $35-40 \%$ & 6.19 & 6.62 & $39.28 \pm 11.3$ & $33.03 \pm 6.8$ & $0.944 \pm 0.27$ & $0.434 \pm 0.19$ & $0.394 \pm 0.179$ & $1.85 \pm 1.48$ & $5.36 \pm 1.41$ \\
\hline $40-45 \%$ & 6.62 & 7.02 & $30.23 \pm 10.2$ & $27.14 \pm 6.66$ & $0.727 \pm 0.24$ & $0.477 \pm 0.202$ & $0.416 \pm 0.187$ & $1.69 \pm 1.39$ & $4.3 \pm 1.34$ \\
\hline $45-50 \%$ & 7.02 & 7.4 & $23.11 \pm 8.95$ & $22.11 \pm 6.43$ & $0.556 \pm 0.22$ & $0.522 \pm 0.213$ & $0.433 \pm 0.193$ & $1.53 \pm 1.29$ & $3.42 \pm 1.25$ \\
\hline $50-55 \%$ & 7.4 & 7.77 & $17.54 \pm 7.79$ & $17.84 \pm 6.08$ & $0.422 \pm 0.19$ & $0.567 \pm 0.22$ & $0.444 \pm 0.199$ & $1.38 \pm 1.18$ & $2.7 \pm 1.15$ \\
\hline $55-60 \%$ & 7.77 & 8.11 & $13.25 \pm 6.69$ & $14.3 \pm 5.65$ & $0.318 \pm 0.16$ & $0.612 \pm 0.225$ & $0.447 \pm 0.208$ & $1.24 \pm 1.07$ & $2.11 \pm 1.04$ \\
\hline $60-65 \%$ & 8.11 & 8.45 & $9.988 \pm 5.67$ & $11.4 \pm 5.13$ & $0.24 \pm 0.14$ & $0.654 \pm 0.228$ & $0.443 \pm 0.22$ & $1.11 \pm 0.954$ & $1.64 \pm 0.922$ \\
\hline $85-90 \%$ & 9.86 & 10.3 & $2.703 \pm 2$ & $3.953 \pm 2.23$ & $0.065 \pm 0.048$ & $0.818 \pm 0.231$ & $0.325 \pm 0.311$ & $0.721 \pm 0.457$ & $0.509 \pm 0.43$ \\
\hline $90-95 \%$ & 10.3 & 11 & $2.116 \pm 1.52$ & $3.261 \pm 1.7$ & $0.0509 \pm 0.037$ & $0.852 \pm 0.223$ & $0.277 \pm 0.319$ & $0.679 \pm 0.377$ & $0.409 \pm 0.36$ \\
\hline $95-100 \%$ & 11 & 19.1 & $1.582 \pm 1.06$ & $2.629 \pm 1.15$ & $0.038 \pm 0.025$ & $0.902 \pm 0.2$ & $0.191 \pm 0.304$ & $0.639 \pm 0.292$ & $0.316 \pm 0.294$ \\
\hline $0-100 \%$ & 0 & 19.1 & $47.93 \pm 59$ & $32.51 \pm 31.7$ & $1.15 \pm 1.4$ & $0.537 \pm 0.3$ & $0.346 \pm 0.233$ & $1.61 \pm 1.54$ & $5.7 \pm 6.2$ \\
\hline
\end{tabular}

[1] M. L. Miller, K. Reygers, S. J. Sanders, and P. Steinberg, Glauber modeling in high energy nuclear collisions, Annu. Rev. Nucl. Part. Sci. 57, 205 (2007).

[2] C. W. De Jager, H. De Vries, and C. De Vries, Nuclear charge and magnetization density distribution parameters from elastic electron scattering, At. Data Nucl. Data Tables 14, 479 (1974).

[3] H. De Vries, C. W. De Jager, and C. De Vries, Nuclear charge and magnetization density distribution parameters from elastic electron scattering, At. Data Nucl. Data Tables 36, 495 (1987).

[4] R. J. Glauber and G. Matthiae, High-energy scattering of protons by nuclei, Nucl. Phys. B 21, 135 (1970).

[5] X.-N. Wang and M. Gyulassy, HIJING: A Monte Carlo model for multiple jet production in $p p, p A$ and $A A$ collisions, Phys. Rev. D 44, 3501 (1991).

[6] B. Alver, M. Baker, C. Loizides, and P. Steinberg, The PHOBOS Glauber Monte Carlo, arXiv:0805.4411.

[7] C. Loizides, J. Nagle, and P. Steinberg, Improved version of the PHOBOS Glauber Monte Carlo, Software X 1, 13 (2015).

[8] W. Broniowski, M. Rybczynski, and P. Bozek, GLISSANDO: Glauber initial-state simulation and more..., Comput. Phys. Commun. 180, 69 (2009).

[9] M. Rybczynski, G. Stefanek, W. Broniowski, and P. Bozek, GLISSANDO 2: GLauber initial-state simulation and more..., ver. 2, Comput. Phys. Commun. 185, 1759 (2014).

[10] C. Loizides, Glauber modeling of high-energy nuclear collisions at the subnucleon level, Phys. Rev. C 94, 024914 (2016).

[11] B. Kłos, A. Trzcińska, J. Jastrzebski, T. Czosnyka, M. Kisieliński, P. Lubiński, P. Napiorkowski, L. Pieńkowski, F. J. Hartmann, B. Ketzer, P. Ring, R. Schmidt, T. von Egidy, R. Smolańczuk, S. Wycech, K. Gulda, W. Kurcewicz, E. Widmann, and B. A. Brown, Neutron density distributions from antiprotonic ${ }^{208} \mathrm{~Pb}$ and ${ }^{209} \mathrm{Bi}$ atoms, Phys. Rev. C 76, 014311 (2007).

[12] C. M. Tarbert et al., Neutron Skin of ${ }^{208} \mathrm{~Pb}$ from Coherent Pion Photoproduction, Phys. Rev. Lett. 112, 242502 (2014).

[13] H. Paukkunen, Neutron skin and centrality classification in highenergy heavy-ion collisions at the LHC, Phys. Lett. B 745, 73 (2015).

[14] S. De, The effect of neutron skin on inclusive prompt photon production in $\mathrm{PbPb}$ collisions at Large Hadron Collider energies, J. Phys. G 44, 045104 (2017).

[15] I. Helenius, H. Paukkunen, and K. J. Eskola, Neutron-skin effect in direct-photon and charged hadron-production in $\mathrm{PbPb}$ collisions at the LHC, Eur. Phys. J. C 77, 148 (2017).

[16] A. Dainese et al., Heavy ions at the Future Circular Collider, CERN Yellow Report 3, 635 (2017).

[17] TGlauberMC on HepForge, version 3.0, http://tglaubermc. hepforge.org/

[18] A. Bialas, M. Bleszynski, and W. Czyz, Multiplicity distributions in nucleus-nucleus collisions at high energies, Nucl. Phys. B 111, 461 (1976).

[19] D. Kharzeev, C. Lourenco, M. Nardi, and H. Satz, A quantitative analysis of charmonium suppression in nuclear collisions, Z. Phys. C 74, 307 (1997).

[20] B. Alver et al. (PHOBOS Collaboration), System Size, Energy, Pseudorapidity, and Centrality Dependence of Elliptic Flow, Phys. Rev. Lett. 98, 242302 (2007).

[21] B. Alver and G. Roland, Collision geometry fluctuations and triangular flow in heavy-ion collisions, Phys. Rev. C 81, 054905 (2010); 82, 039903(E) (2010).

[22] D. Teaney and L. Yan, Triangularity and dipole asymmetry in heavy ion collisions, Phys. Rev. C 83, 064904 (2011). 
[23] B. Alver, B. B. Back, M. D. Baker, M. Ballintijn, D. S. Barton, R. R. Betts, R. Bindel, W. Busza, V. Chetluru, E. Garcia, T. Gburek, J. Hamblen, U. Heinz, D. J. Hofman, R. S. Hollis, A. Iordanova, W. Li, C. Loizides, S. Manly, A. C. Mignerey, R. Nouicer, A. Olszewski, C. Reed, C. Roland, G. Roland, J. Sagerer, P. Steinberg, G. S. F. Stephans, M. B. Tonjes, A. Trzupek, G. J. van Nieuwenhuizen, S. S. Vaurynovich, R. Verdier, G. I. Veres, P. Walters, E. Wenger, B. Wosiek, K. Wozniak, and B. Wyslouch, Importance of correlations and fluctuations on the initial source eccentricity in high-energy nucleus-nucleus collisions, Phys. Rev. C 77, 014906 (2008).

[24] H.-J. Drescher, A. Dumitru, C. Gombeaud, and J.-Y. Ollitrault, The centrality dependence of elliptic flow, the hydrodynamic limit, and the viscosity of hot QCD, Phys. Rev. C 76, 024905 (2007).

[25] A. Drees, H. Feng, and J. Jia, Medium induced jet absorption at RHIC, Phys. Rev. C 71, 034909 (2005).

[26] A. Dainese, C. Loizides, and G. Paic, Leading-particle suppression in high energy nucleus-nucleus collisions, Eur. Phys. J. C 38, 461 (2005).

[27] B. Abelev et al. (ALICE Collaboration), Centrality determination of $\mathrm{PbPb}$ collisions at $\sqrt{s_{N N}}=2.76 \mathrm{TeV}$ with ALICE, Phys. Rev. C 88, 044909 (2013).

[28] D. d'Enterria and T. Pierog, Global properties of proton-proton collisions at $\sqrt{s}=100 \mathrm{TeV}$, J. High Energy Phys. 08 (2016) 170.

[29] G. J. Alner et al. (UA5 Collaboration), Antiproton-proton cross sections at 200 and $900 \mathrm{GeV}$ c.m. energy, Z. Phys. C 32, 153 (1986).

[30] N. A. Amos et al. (E710 Collaboration), A luminosity independent measurement of the $p p$ total cross section at $\sqrt{s}=1.8 \mathrm{TeV}$, Phys. Lett. B 243, 158 (1990).

[31] N. A. Amos et al. (E710 Collaboration), Measurement of $\rho$, the Ratio of the Real to Imaginary Part of the $p^{-} p$ Forward Elastic Scattering Amplitude, at $\sqrt{s}=1.8 \mathrm{TeV}$, Phys. Rev. Lett. 68, 2433 (1992).

[32] F. Abe et al. (CDF Collaboration), Measurement of the $\bar{p} p$ total cross-section at $\sqrt{s}=546 \mathrm{GeV}$ and $1800 \mathrm{GeV}$, Phys. Rev. D 50, 5550 (1994).

[33] F. Abe et al. (CDF Collaboration), Measurement of small angle $\bar{p} p$ elastic scattering at $\sqrt{s}=546 \mathrm{GeV}$ and $1800 \mathrm{GeV}$, Phys. Rev. D 50, 5518 (1994).

[34] B. Abelev et al. (ALICE Collaboration), Measurement of inelastic, single- and double-diffraction cross sections in proton-proton collisions at the LHC with ALICE, Eur. Phys. J. C 73, 2456 (2013).

[35] G. Aad et al. (ATLAS Collaboration), Measurement of the inelastic proton-proton cross section at $\sqrt{s}=7 \mathrm{TeV}$ with the ATLAS Detector, Nat. Commun. 2, 463 (2011).

[36] G. Aad et al. (ATLAS Collaboration), Measurement of the total cross section from elastic scattering in $p p$ collisions at $\sqrt{s}=7 \mathrm{TeV}$ with the ATLAS detector, Nucl. Phys. B 889, 486 (2014).

[37] M. Aaboud et al. (ATLAS Collaboration), Measurement of the total cross section from elastic scattering in $p p$ collisions at $\sqrt{s}=8 \mathrm{TeV}$ with the ATLAS detector, Phys. Lett. B 761, 158 (2016).

[38] M. Aaboud et al. (ATLAS Collaboration), Measurement of the Inelastic Proton-Proton Cross Section at $\sqrt{s}=13 \mathrm{TeV}$ with the ATLAS Detector at the LHC, Phys. Rev. Lett. 117, 182002 (2016).
[39] S. Chatrchyan et al. (CMS Collaboration), Measurement of the inelastic proton-proton cross section at $\sqrt{s}=7 \mathrm{TeV}$, Phys. Lett. B 722, 5 (2013).

[40] H. Van Haevermaet (CMS Collaboration), Measurement of the inelastic proton-proton cross section at $\sqrt{s}=13 \mathrm{TeV}$, in Proceedings of the 24th International Workshop on DeepInelastic Scattering and Related Subjects (DIS 2016): Hamburg, Germany PoS (DIS2016) 198.

[41] R. Aaij et al. (LHCb Collaboration), Measurement of the inelastic pp cross-section at a centre-of-mass energy of $\sqrt{s}=7 \mathrm{TeV}$, J. High Energy Phys. 02 (2015) 129.

[42] G. Antchev et al. (TOTEM Collaboration), First measurement of the total proton-proton cross section at the LHC energy of $\sqrt{s}=7 \mathrm{TeV}$, Europhys. Lett. 96, 21002 (2011).

[43] G. Antchev et al. (TOTEM Collaboration), Luminosityindependent measurements of total, elastic and inelastic crosssections at $\sqrt{s}=7 \mathrm{TeV}$, Europhys. Lett. 101, 21004 (2013).

[44] G. Antchev et al. (TOTEM Collaboration), LuminosityIndependent Measurement of the Proton-Proton Total Cross Section at $\sqrt{s}=8 \mathrm{TeV}$, Phys. Rev. Lett. 111, 012001 (2013).

[45] P. Abreu et al. (Pierre Auger Collaboration), Measurement of the Proton-Air Cross-Section at $\sqrt{s}=57 \mathrm{TeV}$ with the Pierre Auger Observatory, Phys. Rev. Lett. 109, 062002 (2012).

[46] M. Froissart, Asymptotic behavior and subtractions in the Mandelstam representation, Phys. Rev. 123, 1053 (1961).

[47] J. R. Cudell, V. V. Ezhela, P. Gauron, K. Kang, Yu. V. Kuyanov, S. B. Lugovsky, E. Martynov, B. Nicolescu, E. A. Razuvaev, and N. P. Tkachenko (COMPETE Collaboration), Benchmarks for the Forward Observables at RHIC, the Tevatron Run II and the LHC, Phys. Rev. Lett. 89, 201801 (2002).

[48] B. Abelev et al. (ALICE Collaboration), Measurement of the Cross Section for Electromagnetic Dissociation with Neutron Emission in $\mathrm{PbPb}$ Collisions at $\sqrt{s_{N N}}=2.76 \mathrm{TeV}$, Phys. Rev. Lett. 109, 252302 (2012).

[49] V. Khachatryan et al. (CMS Collaboration), Measurement of the inelastic cross section in proton-lead collisions at $\sqrt{s_{N N}}=$ 5.02 TeV, Phys. Lett. B 759, 641 (2016).

[50] B. B. Abelev et al. (ALICE Collaboration), Measurement of visible cross sections in proton-lead collisions at $\sqrt{s_{N N}}=5.02 \mathrm{TeV}$ in van der Meer scans with the ALICE detector, J. Instrum. 9, P11003 (2014).

[51] J. Adam et al. (ALICE Collaboration), Centrality dependence of particle production in $p \mathrm{~Pb}$ collisions at $\sqrt{s_{N N}}=5.02 \mathrm{TeV}$, Phys. Rev. C 91, 064905 (2015).

[52] G. Fricke, C. Bernhardt, K. Heilig, L. A. Schaller, L. Schellenberg, E. B. Shera, and C. W. de Jager, Nuclear ground state charge radii from electromagnetic interactions, At. Data Nucl. Data Tables 60, 177 (1995).

[53] I. Angeli, A consistent set of nuclear rms charge radii: Properties of the radius surface $R(N, Z)$, At. Data Nucl. Data Tables 87, 185 (2004).

[54] I. Angeli and K. P. Marinova, Table of experimental nuclear ground state charge radii: An update, At. Data Nucl. Data Tables 99, 69 (2013).

[55] P. J. Mohr, D. B. Newell, and B. N. Taylor, CODATA recommended values of the fundamental physical constants: 2014, Rev. Mod. Phys. 88, 035009 (2016).

[56] M. Warda, X. Vinas, X. Roca-Maza, and M. Centelles, Analysis of bulk and surface contributions in the neutron skin of nuclei, Phys. Rev. C 81, 054309 (2010). 
[57] S. Wycech, F. J. Hartmann, J. Jastrzebski, B. Klos, A. Trzcinska, and T. von Egidy, Nuclear surface studies with antiprotonic atom X-rays, Phys. Rev. C 76, 034316 (2007).

[58] R. C. Barrett and D. F. Jackson, Nuclear Sizes and Structure (Clarendon Press, Oxford, 1977).

[59] T. Hirano and Y. Nara, Eccentricity fluctuation effects on elliptic flow in relativistic heavy ion collisions, Phys. Rev. C 79, 064904 (2009).

[60] Q. Y. Shou, Y. G. Ma, P. Sorensen, A. H. Tang, F. Videbæk, and $\mathrm{H}$. Wang, Parameterization of deformed nuclei for Glauber modeling in relativistic heavy ion collisions, Phys. Lett. B $\mathbf{7 4 9}$, 215 (2015).

[61] P. Krishna and D. Pandey, Close-Packed Structures (University College Cardiff Press, Cardiff, Wales, 1981).

[62] M. Alvioli, H. Holopainen, K. J. Eskola, and M. Strikman, Initial state anisotropies and their uncertainties in ultrarelativistic heavy-ion collisions from the Monte Carlo Glauber model, Phys. Rev. C 85, 034902 (2012).

[63] M. Alvioli, H. J. Drescher, and M. Strikman, A Monte Carlo generator of nucleon configurations in complex nuclei including nucleon-nucleon correlations, Phys. Lett. B 680, 225 (2009).

[64] J. T. Mitchell, D. V. Perepelitsa, M. J. Tannenbaum, and P. W. Stankus, Tests of constituent-quark generation methods which maintain both the nucleon center of mass and the desired radial distribution in Monte Carlo Glauber models, Phys. Rev. C 93, 054910 (2016).

[65] D. Miskowiec, Nuclear Overlap Calculation, http://web-docs. gsi.de/misko/overlap/; code adapted to match the MC implementation as closely as possible.

[66] R. Hofstadter, Electron scattering and nuclear structure, Rev. Mod. Phys. 28, 214 (1956).

[67] M. Alvioli and M. Strikman, Color fluctuation effects in protonnucleus collisions, Phys. Lett. B 722, 347 (2013).

[68] R. Corke and T. Sjostrand, Multiparton interactions with an xdependent proton size, J. High Energy Phys. 05 (2011) 009.

[69] M. Rybczyński and Z. Włodarczyk, The nucleon-nucleon collision profile and cross section fluctuations, J. Phys. G: Nucl. Part. Phys. 41, 015106 (2013).

[70] J. Jia, Influence of the nucleon-nucleon collision geometry on the determination of the nuclear modification factor for nucleonnucleus and nucleus-nucleus collisions, Phys. Lett. B 681, 320 (2009).

[71] C. Loizides and A. Morsch, Absence of jet quenching in peripheral nucleus-nucleus collisions, Phys. Lett. B 773, 408 (2017).

[72] R. Brun and F. Rademakers, ROOT: An object oriented data analysis framework, Nucl. Instrum. Methods A 389, 81 (1997).

[73] K. Tsukada et al., First Elastic Electron Scattering from ${ }^{132} \mathrm{Xe}$ at the SCRIT Facility, Phys. Rev. Lett. 118, 262501 (2017). 